\title{
UN MODELO INTERDISCIPLINARIO PARA LA MACROECONOMÍA*
}

\author{
Andrew G. Haldane \\ Arthur E. Turrell
}

" DOI: https://doi.org/10.18601/01245996.v21n40.03. Traducido con el consentimiento del Banco de Inglaterra de la versión original titulada An interdisciplinary model for macroeconomics, Bank of England staff working paper 696 [https://www.bankofengland.co.uk/working-paper/2017/an-interdisciplinary-model-for-macroeconomics], con derechos de autor del Banco de Inglaterra, que no revisó la traducción y no garantiza su exactitud ni su integridad. Cualquier reproducción está sujeta al consentimiento del Banco de Inglaterra y de Revista de Economía Institucional. Traducción de Alberto Supelano. Recepción: 10-08-2018, aceptación: 30-10-2018. Sugerencia de citación: Haldane, A. G. y Turrell A. E. (2019). Un modelo interdisciplinario para la macroeconomía. Revista de Economía Institucional, 21(40), 69-111.

a Banco de Inglaterra, [andy.haldane@bankofengland.co.uk].

bBanco de Inglaterra, [arthur.turrell@bankofengland.co.uk], [https://orcid. org/0000-0002-2525-0773] 


\section{Un modelo interdisciplinario para la macroeconomía}

Resumen. La modelación macroeconómica está bajo intenso escrutinio desde la gran crisis financiera, que dejó al descubierto los graves defectos de la metodología utilizada para entender la economía en su conjunto. Se critican los supuestos empleados en los modelos dominantes, en particular que los agentes económicos son homogéneos y optimizadores y que la economía se equilibra. Este escrito explora un enfoque interdisciplinario de modelación macroeconómica con técnicas tomadas de otras ciencias, y examina la modelación basada en agentes como ejemplo de esas técnicas. Los modelos basados en agentes complementan los enfoques existentes y son adecuados para responder preguntas macroeconómicas donde la complejidad, la heterogeneidad, las redes y las heurísticas cumplen un papel importante.

Palabras clave: macroeconomía, modelación, modelo basado en agentes; JEL: E17, E60, C60, A12.

\section{An interdisciplinary model for macroeconomics}

Abstract. Macroeconomic modelling has been under intense scrutiny since the Great Financial Crisis, when serious shortcomings were exposed in the methodology used to understand the economy as a whole. Criticism has been levelled at the assumptions employed in the dominant models, particularly that economic agents are homogeneous and optimising and that the economy is equilibrating. This paper seeks to explore an interdisciplinary approach to macroeconomic modelling, with techniques drawn from other (natural and social) sciences. Specifically, it discusses agent-based modelling, which is used across a wide range of disciplines, as an example of such a technique. Agent-based models are complementary to existing approaches and are suited to answering macroeconomic questions where complexity, heterogeneity, networks, and heuristics play an important role.

Keywords: Macroeconomics, modelling, agent-based model; JEL: E17, E60, C60, A12.

\section{Um modelo interdisciplinar para a macroeconomia}

Resumo. A modelagem macroeconômica está sob intensa análise desde a grande crise financeira, que expôs as graves falhas da metodologia utilizada para entender a economia como um todo. As premissas utilizadas nos modelos dominantes são criticadas, em particular, que os agentes econômicos são homogêneos e otimizadores, e que a economia é equilibrada. Este artigo explora uma abordagem interdisciplinar da modelagem macroeconômica com técnicas emprestadas de outras ciências e examina a modelagem baseada em agentes como um exemplo dessas técnicas. Os modelos baseados em agentes complementam as abordagens existentes e são adequados para responder a questões macroeconômicas em que complexidade, heterogeneidade, redes e heurísticas desempenham um papel importante.

Palavras-chaves: macroeconomia, modelagem, modelo baseado em agentes; JEL: E17, E60, C60, A12. 
T a crisis económica y financiera generó una crisis en la profesión Leconómica y financiera. Algo similar ocurrió después de la Gran Depresión cuando se replanteó la economía bajo el liderazgo intelectual de Keynes (1936). El reto para los académicos y las autoridades de política es hoy semejante. Se han puesto en cuestión los modelos utilizados antes de la crisis. Los críticos argumentan que eran demasiado restrictivos y no tenían respaldo empírico. El trabajo académico empieza a llenar esos vacíos y se buscan nuevas perspectivas. Asumir el reto de modelar un conjunto más amplio de circunstancias económicas requiere que los macroeconomistas piensen de una nueva manera, quizá inspirándose en otras disciplinas con enfoques metodológicos diferentes. Este escrito describe las dificultades que enfrenta la modelación macroeconómica, examina cuán beneficiosa podría ser una metodología más pluralista y sugiere un camino constructivo para enfrentar esos retos.

La primera sección presenta evidencia de la insularidad en economía y considera por qué puede no ser óptima. La segunda discute esta "monocultura macroeconómica", quizá mejor ilustrada por el predominio del marco del agente representativo que opera con expectativas racionales; aboga por un conjunto más diverso de modelos macroeconómicos, incluidos los que experimentan con diferentes comportamientos de los agentes. La tercera describe los orígenes interdisciplinarios del enfoque basado en agentes. La cuarta contrasta la filosofía de la modelación basada en agentes con otros enfoques de modelación macroeconómica. La quinta pregunta qué pueden hacer los modelos basados en agentes por la macroeconomía y la última presenta algunas conclusiones.

\section{¿UNA DISCIPLINA INSULAR?}

La economía, en particular la macroeconomía, ha sido históricamente insular como disciplina, al menos en comparación con otros campos. Esta no es una observación nueva; ya ha sido señalada (p. ej., en Hausman, 1992). Y, pese al avance reciente, hay evidencia que sugiere que la gran crisis financiera no disipó del todo esta insularidad.

La gráfica 1 muestra que, entre 1950 y 2010, los artículos de economía en revistas académicas citaron artículos de otras disciplinas con una frecuencia menor que el promedio. A su vez, esas otras disciplinas no citaron consistentemente la investigación económica. Esto sugiere que las nuevas ideas no fluyeron libremente, dentro o fuera de la economía. La insularidad es en parte una consecuencia 
natural de la estratificación, debido a que las disciplinas académicas existen como áreas de investigación parcialmente reservadas donde los especialistas desarrollan y centran su atención (Jacobs, 2014). Pero parece ser peculiarmente intensa en economía, como reportan Fourcade et al. (2015).

Gráfica 1

Citas dentro y fuera de las disciplina, por disciplinas

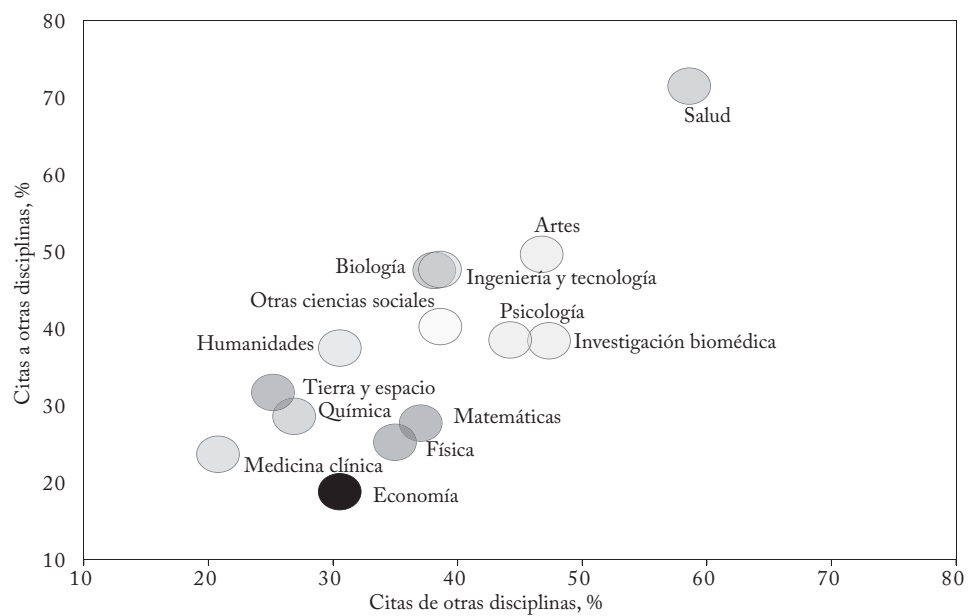

Citas a otras disciplinas, \% Citas de otras disciplinas, \%

Salud Artes Biología Ingeniería y tecnología Otras ciencias sociales Humanidades Psicología Investigación biomédica Tierra y espacio Química Matemáticas Física Medicina clínica Economía Fuente: Van Noorden (2015).

Esta situación ha mejorado en los últimos años, como muestra la gráfica 2. No obstante, la economía sigue siendo más autorreferencial que muchos otros campos, sin duda menos interdisciplinarios. Incluso las matemáticas, a las que muchos consideran un modelo de "pureza", están por encima de la economía en la gráfica 2. E1 trabajo interdisciplinario implica costos adicionales (Yegros et al., 2015), en particular los que surgen de la coordinación entre áreas de estudio, de las revistas más respetadas que tienen un sesgo natural hacia ese campo y de las dificultades de una revisión precisa de los temas. Pero no hay ninguna razón para suponer que estos factores son más agudos en economía que en otras disciplinas.

Además de esta evidencia de insularidad interdisciplinaria, hay evidencia de mayor insularidad intradisciplinaria. El número de autores por artículo en economía es menor que en otras disciplinas, como muestra la gráfica 3. El fenómeno de la "gran ciencia" ha aumentado los rendimientos a escala de la colaboración académica en 
los últimos años, de científicos que se reúnen en equipos cada vez más grandes, que a veces colaboran durante varias generaciones, para lograr los avances más espectaculares (Aad et al., 2012; Abbott et al., 2016). Wuchty et al. (2007) muestran que la investigación de equipos más grandes se cita más, en promedio, que la investigación de un solo autor, en todas las disciplinas. Curiosamente, eso también es cierto para las cinco principales revistas de economía, donde un artículo de cuatro autores es un 61\% más citado que un artículo de un solo autor (Card y DellaVigna, 2013).

Gráfica 2

Numero de citas de la disciplina que se muestra a otras disciplinas

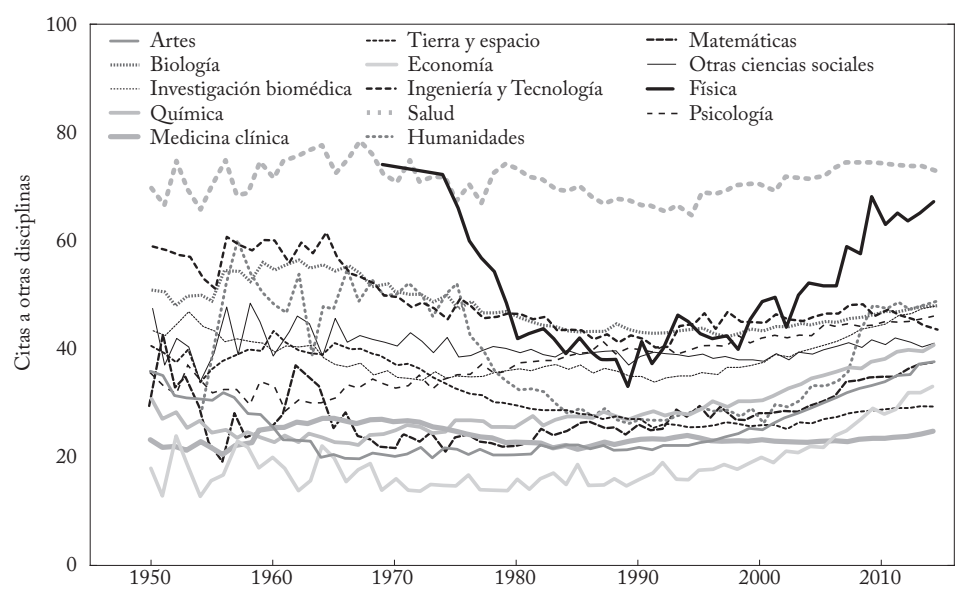

Fuente: Van Noorden (2015).

A pesar de estos beneficios, la mayoría de las ciencias naturales están por encima de la economía en medidas de insularidad intradisciplinaria. A medida que crece el tamaño de las bases de datos disponibles para los macroeconomistas, el trabajo en equipos pequeños puede perder sustanciales economías de escala en la investigación. Y los experimentos, para algunas personas el patrón oro del método científico, se asocian a un aumento del número de autores por artículo (Hamermesh, 2013).

La falta de diversidad es otra señal de insularidad. Un estudio reciente encontró que el $43 \%$ de los artículos publicados en las cuatro principales revistas de economía fue escrito por académicos conectados con uno de los editores en el momento de su publicación (Colussi, 2018). Las autoras representaron menos del 15\% de los artículos de las tres principales revistas de economía en 2011. ¿Esto es importante 
para la calidad de la investigación económica? Se suele pensar que la diversidad es buena para la innovación (Carney, 2017; Page, 2008). Y los riesgos de lo opuesto, una monocultura de investigación, pueden ser graves (Bronk, 2011; Bronk y Jacoby, 2016).

Gráfica 3

Número promedio de autores por artículo

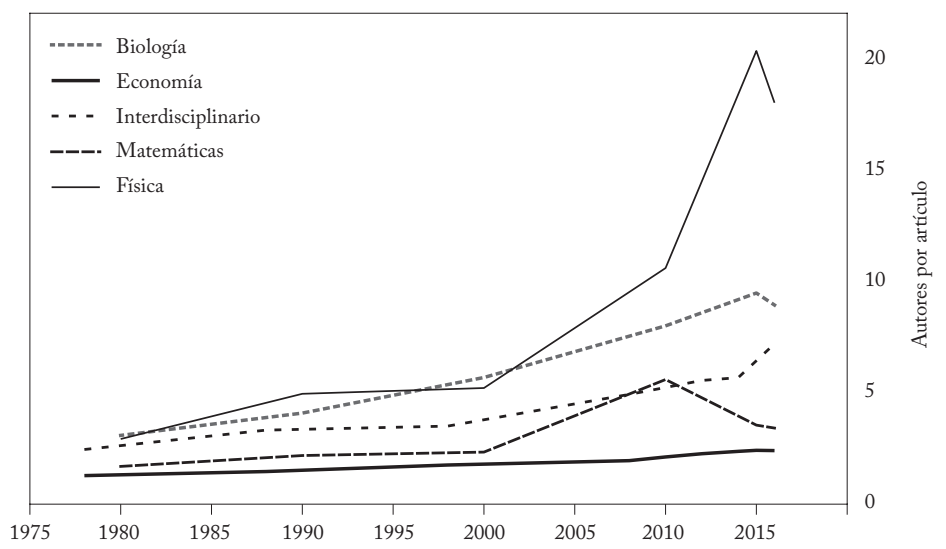

Número promedio de autores por artículo de los 20.000 principales artículos por número de citas en cada categoría de temas de Scopus por cada año, años y temas seleccionados.

Fuente: Scopus.

Es curioso que la evidencia más sólida de esto provenga de los mismos economistas. En 2006, cuando se les preguntó si estaban de acuerdo o en desacuerdo con la afirmación "En general, el conocimiento interdisciplinario es mejor que el conocimiento obtenido por una sola disciplina”, cerca del 60\% de los economistas académicos dijo que estaba en gran desacuerdo contra un promedio del $21 \%$ en sociología, ciencia política, psicología, finanzas e historia (Fourcade et al., 2015).

La macroeconomía es quizá la subdisciplina más visible para el público en general. Sin embargo, en el Reino Unido, casi todos los grupos de la sociedad confían mucho más en los científicos (gráfica 4a) que en los economistas (gráfica 4b). Esto tiene muchas causas posibles. No lo explica el mayor contenido técnico de la economía. Lo podría explicar, al menos en parte, la manera de comunicar ese contenido técnico al público lego. Esto, a su vez, puede generar falta de confianza en los modelos y métodos de los economistas, de una manera que no se aplica a los científicos.

No obstante, debido precisamente a que la economía combina elementos de las ciencias naturales y sociales, sus puntos de tangencia disciplinaria tal vez son mayores que en muchos otros 
campos de estudio. La economía tiene largo alcance. Como dijo Keynes (1924):

el buen economista debe poseer una rara combinación de dones [...] Debe ser, en cierta medida, matemático, historiador, estadista y filósofo. Debe entender símbolos y expresarse en palabras. Debe contemplar lo particular en términos de lo general y tocar lo abstracto y lo concreto en el mismo vuelo de pensamiento. Debe estudiar el presente a la luz del pasado para los propósitos del futuro. Ninguna parte de la naturaleza del hombre o de sus instituciones debe ser totalmente ajena a su consideración.

Gráfica 4

Confianza de red para diferentes agrupamientos

[a) Confianza...

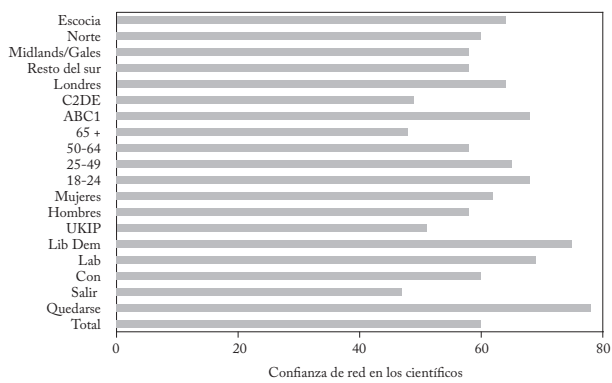

b) Confianza...]

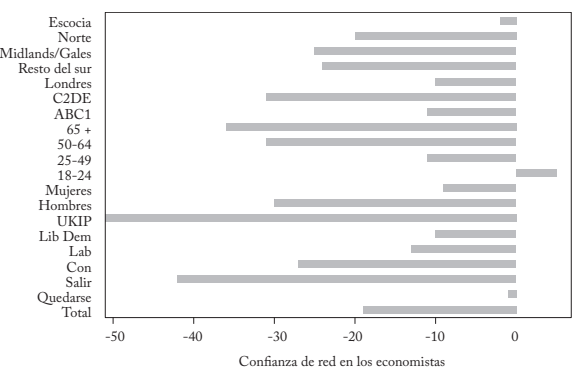

Los datos que se muestran son los resultados de una encuesta a 2.040 adultos británicos del 14 al 15 de febrero 2017. "Salir" y "Quedarse" se refieren a la elección de los que votaron en el referéndum del Reino Unido sobre la pertenencia a la Unión Europea.

Fuente: YouGov.

La descripción de Keynes del economista es tan relevante hoy como lo era en 1924, aunque en estos días podríamos añadir a la lista: estadístico, científico de computación, psicólogo e incluso biólogo evolutivo. La macroeconomía tiene mucho que ganar inspirándose en otras disciplinas; y otras disciplinas podrían beneficiarse, a su vez, de una mejor comprensión de la economía (Stern, 2016a), incluidas las técnicas de modelación macroeconómica (Tasoff et al., 2015).

Algunos ejemplos pueden ilustrar algunas de las áreas potencialmente fértiles habitadas recientemente por la investigación interdisciplinaria en economía. En epidemias recientes, los mayores costos económicos no provienen de los efectos directos de las muertes, sino de cambios en el comportamiento de las personas como respuesta (Avian Flu Working Group, 2006; Keogh et al., 2010; Sands et al., 2016). Eso mismo se puede decir del daño colateral a la economía causado por recesiones y crisis. Por ejemplo, los modelos epidemiológicos son prometedores para explicar el contagio financiero en 
épocas de crisis financiera (Arinaminpathy et al., 2012; Haldane y May, 2011).

Un segundo ejemplo es la tecnología y la innovación. Existen muchos ejemplos de tecnologías transformadoras que pueden tener efectos macro. Los mercados digitales están mejorando los procesos de emparejamiento y reduciendo la asimetría de información. Los "grandes datos" prometen identificar nuevos factores de riesgo, mientras que la inteligencia artificial y la robótica ofrecen sustanciales ganancias de productividad. También existen riesgos: los algoritmos pueden coludirse acerca del precio u operar de manera prejuiciada (Ezrachi y Stucke, 2016). Los métodos de análisis de textos, con ayuda de nuevos algoritmos como Word2Vec (Mikolov et al., 2013), están mejorando los pronósticos macroeconómicos (Baker et al., 2016; Nyman et al., 2015). La comprensión de las ciencias de la información y la capacidad para usar técnicas informáticas son cada vez más útiles para los economistas.

Un tercer ejemplo es el cambio climático. E1 Banco de Inglaterra hace poco publicó una investigación sobre algunas de las implicaciones del cambio climático (Batten et al., 2016). El efecto de la temperatura global sobre la productividad, el crecimiento y la estabilidad financiera es altamente no lineal y fuertemente negativo más allá de un umbral de temperatura (Burke et al., 2015). Para entender esos efectos macroeconómicos es necesario combinar conocimientos de las ciencias naturales y sociales.

Un cuarto ejemplo, quizá el más exitoso, es la economía del comportamiento: la fusión de la psicología y la economía de la elección (Tversky y Kahneman, 1975). Esta replantea la visión de la manera como los agentes económicos toman decisiones y puede ayudar a configurar modelos de la macroeconomía y la política macroeconómica. Quizá ya haya ayudado a elevar el lugar de la economía en la gráfica 2. Pero aún falta camino por recorrer.

\section{LA MONOCULTURA MACROECONÓMICA}

¿Por qué la macroeconomía se volvió insular? En economía política se tenía una visión mucho más amplia, así como la tenían muchos economistas del siglo XIX y comienzos del XX. El predominio de una sola metodología en esta área puede ofrecer una clave. Este tuvo origen en la "contrarrevolución nueva clásica" de la década de 1970, en la que Lucas, Sargent, Kydland y Prescott (Kydland y Prescott, 1982; Lucas y Sargent, 1979) derrocaron el uso de la modelación 
"estructural" o de "política" de variables macroeconómicas agregadas. Esos modelos econométricos estructurales, dijeron Lucas y otros, padecían varios errores, como decir poco de la estanflación de los años setenta y adoptar supuestos teóricos inverosímiles para que los datos coincidieran.

La crítica más conocida de Lucas a estos modelos fue que no eran robustos a cambios de política, que no admitían que el comportamiento de los agentes cambiara cuando cambiaban los incentivos de esos agentes (Lucas, 1976). Esta crítica es razonable en cuanto los modelos robustos deberían tratar de explicar de qué modo los cambios de política pueden afectar los resultados agregados. Pero, en la práctica, ningún modelo pasa del todo la prueba-crítica de Lucas; es una cuestión de grado.

Lucas y otros desarrollaron una metodología que creían que no sería afectada por la crítica. El paradigma en que creía Lucas era basar estas fluctuaciones macroeconómicas en lo que se conoce como "microfundamentos". En pocas palabras, que el comportamiento macroeconómico se debía construir agregando las acciones individuales de agentes optimizadores que buscan su interés personal. Al basarse en el comportamiento optimizador estos comportamientos egoístas eran menos susceptibles de cambiar cuando cambiaban las relaciones macroeconómicas agregadas.

En la práctica, tales microfundamentos a menudo se asociaban a un tipo particular de comportamiento: la optimización con expectativas racionales (Lucas, 1972, 1987; Muth, 1961). La forma débil de expectativas racionales es la siguiente: sea $I_{t-1, i}$ el conjunto de información disponible para el agente $i$ al comienzo del periodo $t$. Este agente tiene una expectativa individual $\mathbb{E}_{t-1, i}\left\{p_{t}+s\right\}$ para el valor de $p$ en $t+s$, donde $s \geq 0$. Se define $\mathbb{E}_{t-1, i}\left\{p_{t+s} \mid I_{t-1, i}\right\}$ como la expectativa verdadera de $p_{t+s}$ dada la información disponible. La forma débil de expectativas racionales es definida entonces por:

$\mathbb{E}_{t-1, i}\left\{p_{t+s}\right\}=\mathbb{E}_{t-1, i}\left\{p_{t+s} \mid I_{t-1, i}\right\}+\varepsilon_{t-1, i}, \quad \mathbb{E}_{t-1, i}\left\{\varepsilon_{t-1, i} \mid I_{t-1, i}\right\}=0$

donde $\varepsilon_{i, t}$ es un término de error. Nótese que para que esta forma débil sea verdadera, debe existir la distribución de probabilidad objetiva $f$, donde $p \sim f$. La forma más fuerte de expectativas racionales, tal como se especifica en Muth (1961), añade a la forma débil los supuestos de que cada agente conoce el comportamiento y las decisiones que tomarán todos los demás agentes, los valores verdaderos de todos los parámetros exógenos deterministas que rigen la evolución de la economía, las propiedades de todas las distribuciones de probabilidad 
que rigen las variables exógenas estocásticas (como f), y los valores realizados de las variables endógenas. A estas expectativas se acoplan los supuestos sobre optimalidad del agente, normalmente suponiendo que los agentes maximizan la suma descontada de sus utilidades futuras esperadas, sujeta a una restricción presupuestal.

Estos microfundamentos tienen varias propiedades deseables. Pueden servir como aproximación útil del comportamiento del mundo real, al menos en algunas situaciones. Condensan el mundo en un pequeño número de factores fácilmente observables, que pueden ayudar a determinar qué impulsa qué. $\mathrm{Y}$ a veces son más manejables y más elegantes analíticamente que la mayoría de las alternativas.

Pero tienen varias limitaciones. Por un lado, invocan supuestos fuertes (como expectativas racionales y optimización) que los datos a menudo no confirman (Estrella y Fuhrer, 2002). Una defensa de las expectativas racionales es que, si bien los individuos pueden no ser racionales, sus irracionalidades se cancelan a nivel agregado. Shaikh (2016) muestra que varios comportamientos microeconómicos diferentes pueden llevar a iguales resultados macroeconómicos. Pero es bien sabido a partir de otras disciplinas que, en general, el comportamiento heterogéneo a nivel micro se combina para generar respuestas complejas no lineales y un comportamiento emergente a nivel macro. Hay también escenarios simples en los que se derrumba la versión fuerte de las expectativas racionales, por ejemplo, en juegos de minorías (Arthur, 2006). En general, hay relativamente pocos casos en los que sea totalmente sólida la agregación a nivel macro emprendida en modelos que usan expectativas racionales (Kirman, 1992).

Un conjunto alternativo plausible de microfundamentos se basaría, en cambio, en comportamientos observados empíricamente de consumidores, empresas y gobiernos. Los comportamientos observados se suelen denominar heurísticas o "reglas generales". A menudo provienen de modelos que toman ideas de la psicología para entender el comportamiento humano (Tversky y Kahneman, 1975). Lo que constituye racionalidad no es claro en sí mismo ni está bien definido (Simon, 1959). De hecho, en un mundo de incertidumbre knightiana (Knight, 2012), información imperfecta, altruismo y computación costosa, hay un cuerpo emergente de evidencia que sugiere que las heurísticas son más "racionales", al menos medidas por el desempeño, que la racionalidad en sentido estrecho, en la que los agentes calculan el curso de acción óptimo sin limitaciones a su capacidad de reunir o procesar información (Aikman et al., 2014; Assenza et al., 2017; Gode y Sunder, 1993; Haldane y Madouros, 2012; Hommes, 2006). Esto a 
veces se llama "racionalidad ecológica" de las heurísticas (Gigerenzer y Brighton, 2009).

Los modelos que dominan la metodología de expectativas racionales con optimización en macroeconomía son los modelos dinámicos estocásticos de equilibrio general (DSGE) (Smets y Wouters, 2003). En su forma más reducida, estos modelos tienen un equilibrio único con pequeñas y suaves desviaciones, sin ningún papel de las variables de nivel y del comportamiento micro de los agentes, que se puede agregar simple y linealmente en el comportamiento de un agente representativo con expectativas racionales. La mayoría de los bancos centrales adoptan el marco DSGE como punto de partida, incluido el Banco de Inglaterra (Burgess et al., 2013).

Ha habido mucho debate sobre los pros y contras de los modelos DSGE que no repetimos aquí (Colander et al., 2009; Fair, 2012; Smith, 2014). Una crítica muy sólida es que sus adiciones se parecen a los epiciclos del sistema astronómico de Ptolomeo (Fagiolo y Roventini, 2012, 2017), que sus agentes representativos son "Robinson Crusoes estocásticos" (Summers, 2002) y que en conjunto son "post reales" (Romer, 2016). Después de la crisis, los investigadores han añadido heterogeneidad de tipos de agentes, un papel del sector financiero y racionalidad limitada de algunos agentes. Algunos modelos usan choques exógenos con distribuciones de colas gordas en vez de distribuciones gaussianas (Ascari et al., 2015). Todas estas adiciones son útiles. Existen modelos anteriores de expectativas racionales con heterogeneidad añadida por medio de funciones de densidad (ver, p. ej., Heathcote, 2005). Los creadores del modelo DSGE usual antes de la crisis (Smets y Wouters, 2003) hace poco publicaron un artículo que contiene muchas de estas modificaciones, incluido un límite inferior igual a 0 , choques no gaussianos y un acelerador financiero. Encuentran que las extensiones.

en cierto modo explican las características de la Gran Recesión y sus consecuencias, pero no son suficientes para abordar algunos de los principales retos de política asociados al uso de una política monetaria y unas políticas macroprudenciales no convencionales (Lindé et al., 2016).

También existen nuevas direcciones de investigación, como los modelos DSGE nuevo keynesianos de agentes heterogéneos (Kaplan et al., 2016; Ravn y Sterk, 2016), que presentan más de un equilibrio.

A pesar de estas importantes modificaciones, parece probable que algunas características de los sistemas económicos sigan siendo muy difíciles de reproducir en un esquema DSGE, como la dinámica de las crisis. Los modelos DSGE han intentado explicar simultánea- 
mente los hechos estilizados observados en sistemas económicos reales (Fukac y Pagan, 2006). El cuadro 1 muestra una selección de tales hechos estilizados. Un ejemplo importante es la distribución del crecimiento del PIB observada históricamente que se muestra en la gráfica 5 . Cerca del 18\% de los datos del Reino Unido, Estados Unidos, Alemania y Japón caen fuera de la distribución normal mejor ajustada. Los modelos DSGE no tienden a reproducir, excepto por construcción, la variación excesivamente grande del crecimiento del PIB observada en los datos históricos (Ascari et al., 2015).

Gráfica 5

Distribución del crecimiento del PIB año a año, 1871-2015

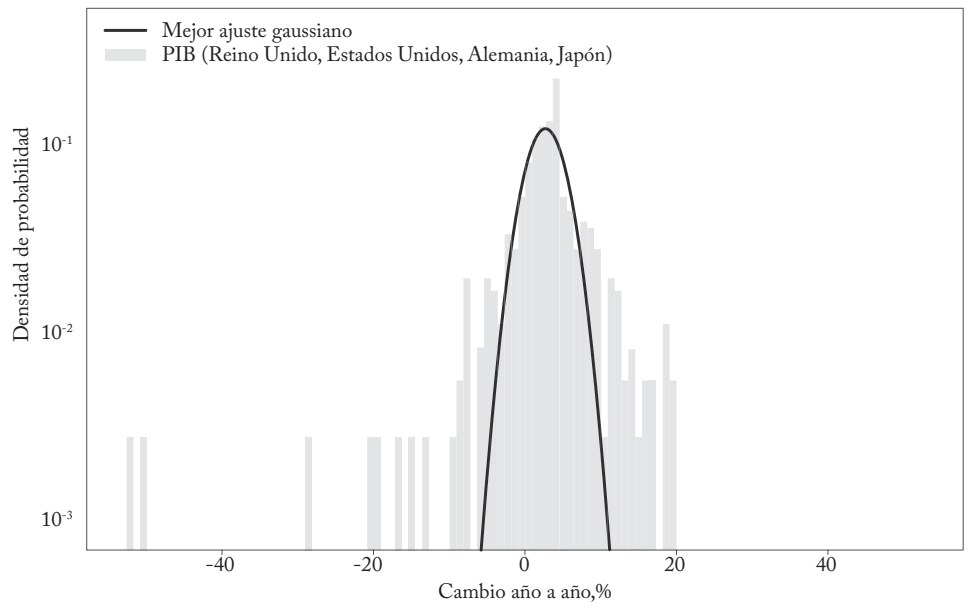

Fuente: Hills et al. (2016).

La crisis financiera global da un buen ejemplo de estas limitaciones. Los modelos DSGE intentaron explicar cómo empezó o cómo se propagó. La gráfica 6 muestra el rango de pronósticos del crecimiento del PIB del Reino Unido realizados por 27 pronosticadores económicos (incluido el Banco) en 2007. Los pronósticos anteriores a la crisis se agruparon estrechamente en un rango de un punto porcentual. La monocultura metodológica produjo, como era de esperar, el mismo resultado. Estos pronósticos previeron una continuación de las suaves ondulaciones observadas en la década anterior a la crisis, la llamada Gran Moderación (Bemanke, 2004). En ese momento, las oscilaciones amortiguadas de la Gran Moderación parecían ajustarse bien al suave movimiento de los modelos DSGE. 
Cuadro1

Ejemplos de hechos estilizados

Hecho estilizado Ejemplos

Crecimiento endógeno autosostenido Burns y Mitchell (1946), Kuznets y Murphy (1966), Stock y Watson (1999), Zarnowitz (1985)

Distribución de la tasa de crecimiento Castaldi y Dosi (2009), Fagiolo et al. (2008)

del PIB de cola gorda

Duración de la recesión distribuida exponen- Ausloos et al. (2004), Wright (2005)

cialmente

Volatilidad relativa del PIB, el consumo y la inversión

Correlaciones cruzadas de variables macro

Inversión agregada procíclica en investigación y desarrollo

Correlaciones cruzadas de variables relacionadas con el crédito

Correlación cruzada entre deuda y pérdidas de préstamos de la firma

La distribución de la duración de las crisis

bancarias es sesgada a la derecha

La distribución de la relación entre costos

fiscales de las crisis bancarias y PIB es de

cola gorda

La distribución del tamaño de la firma $(\log )$

es sesgada a la derecha

La distribución de la tasa de crecimiento de

la firma es de cola gorda

Heterogeneidad de la productividad entre

firmas

Persistente diferencial de productividad entre Bartelsman y Doms (2000), Dosi (2007)

firmas

Tasas de inversión "bruta" a nivel de firma

Contraciclicidad de las bancarrotas de las

firmas

La distribución de la mala deuda de las

Doms y Dunne (1998)

Napoletano et al. (2006), Stock y Watson

(1999)

Napoletano et al. (2006), Stock y Watson (1999)

Walde y Woitek (2004)

Leary (2009), Lown y Morgan (2006)

Foos et al. (2010), Mendoza y Terrones

(2012)

Reinhart y Rogoff (2009)

Laeven y Valencia (2013)

Dosi y Bottazzi et al. (2007)

Bottazzi y Secchi $(2003,2006)$

Bartelsman y Doms (2000), Dosi (2007)

firmas se ajusta a una ley de potencias

Los tamaños de firma se ajustan a una ley de Gaffeo et al. (2012) potencias de Taylor

E1 punto más importante aquí no es que estos modelos no predijeran el momento preciso de la crisis. Casi por definición, las crisis financieras costosas no se pueden predecir porque, si se pudiese, los bancos centrales y los gobiernos tomarían medidas para evitarlas. El problema real fue que nada decían de la probabilidad de que surgiera endógenamente una crisis grave en algún momento, ni de las consecuencias para la economía de una crisis una vez se produjera. La ausencia de expectativas no racionales, de heurísticas y canales de amplificación no lineales fue quizá clave para explicar estos problemas. 
Gráfica 6

Rango de pronósticos del PIB en IV-2007

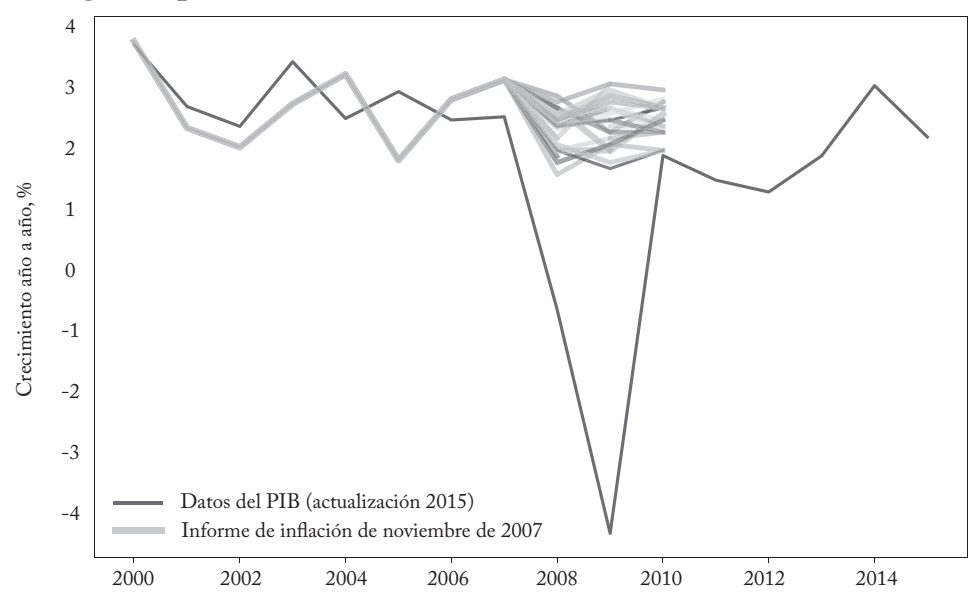

Fuente: Haldane (2016).

El punto más general es que es improbable que un solo modelo marco satisfaga mejor las necesidades de los macroeconomistas en todo estado de la naturaleza. En el mejor de los casos, el método científico exige aplicar experimentos cuidadosamente controlados a una variada ecología de modelos, para que sea posible la selección gradual de los que mejor se ajustan a los hechos conocidos. Pero los macroeconomistas rara vez tienen el lujo de hacer experimentos. E incluso cuando pueden, es difícil validar un modelo experimental porque la macroeconomía es un sistema complejo de partes que interactúan en el que es difícil separar causas y efectos.

Ante estas restricciones, es probable que un mosaico de modelos sea más elástico que una sola metodología. Es posible que un grupo de modelos auténticamente distintos, que compitan para ajustarse a los momentos del mundo real, produzca un conjunto de percepciones mucho más fructífero que un solo tipo de modelos, por estéticamente bello que sea. En otras palabras, quizá se necesite una "explosión cámbrica" en la modelación macroeconómica.

A partir de los pronósticos, hay evidencia de que la combinación de dos o más modelos lleva a un mayor poder de predicción que el uso de un solo modelo (Silver, 2012; Stock y Watson, 2006; Timmermann, 2006). Así lo demostraron Bjørnland et al. (2012) para el pronóstico de inflación del Norges Bank. Es posible que sea cierto cualitativa y cuantitativamente; lo que no capta ni explica bien un modelo puede ser explicado por otro. Un conjunto de modelos distintos, pero plausibles, 
será más informativo en conjunto, en especial cuando no concuerdan. Este enfoque de "zoo de modelos" también se adoptó en el Banco de Inglaterra (Burgess et al., 2013).

¿Qué tipos de animales pueden ser más útiles en el zoológico? A un alto nivel, hay dos tipos: modelos económicos "tipo I" de un solo equilibrio para abordar fluctuaciones cercanas al equilibrio, y modelos económicos más complejos "tipo II" de equilibrios múltiples para abordar fluctuaciones alejadas del equilibrio. Los modelos tipo I son estacionarios y aproximadamente lineales. Los comportamientos son bien anclados y cercanos a la optimización. Las interacciones de los agentes son predecibles y se agregan a algo cercano a un solo agente representativo (Kirman, 1992; Solow, 2008).

Los modelos tipo II captan comportamientos que son "irracionales" o "heurísticamente racionales" y heterogéneos. La incertidumbre, distinta del riesgo, es aguda. Es probable que el comportamiento agregado en estos modelos sea de cola gorda y a menudo emergente. Los modelos tipo II también pueden ayudar a educarnos representando escenarios que no esperábamos al construir el modelo, combinando características conocidas de nivel micro para producir resultados agregados inesperados. Esta es la filosofía "si no lo cultivaste, no lo explicaste" descrita en Epstein (1999).

Los modelos macroeconómicos tipo II deberían ser capaces de explicar cómo pueden surgir endógenamente características económicas como las crisis. Para dar un ejemplo concreto, un análisis tipo I de la crisis invocaría un choque exógeno de crisis -en el que un gran número de consumidores incumple una fracción significativa de sus préstamos- para entender cómo evolucionó después la gran crisis financiera (Kumhof et al., 2015). Un modelo tipo II mostraría que las crisis no aparecen debido a un choque exógeno, sino como una consecuencia natural de las reglas y comportamientos de los agentes dentro del modelo del sistema económico en el tiempo. Esto proporciona un mecanismo para explorar políticas que reduzcan la frecuencia o gravedad de las crisis.

Como señalaron Krugman y otros autores (Colander et al., 2009; Krugman, 2011), algunas formas parciales de (des)equilibrio de concebir el sistema económico podrían haber ayudado a entender la crisis antes de que se desarrollara, incluidas las ideas de Bagehot (1873), Leijonhufvud (2000), Kindleberger (2001) y Minsky (2008). Antes de la contrarrevolución nueva clásica, también eran populares los modelos econométricos estructurales (Blanchard, 2017); el modelo macro FRB/US de la Reserva Federal de Estados Unidos entra en 
esta categoría (Brayton y Tinsley, 1996; ver Welfe, 2013 para una revisión de este tipo de modelos). Pero todos ellos tienden a operar a nivel agregado y en equilibrio general, en vez de agregar a partir del nivel de agentes. Por eso son menos adecuados para abordar problemas con alto grado de heterogeneidad de agentes o con equilibrios cambiantes.

La hegemonía del enfoque del "agente representativo con expectativas racionales" está muy arraigada en macroeconomía. Tiene semejanzas con la física newtoniana. Pero aunque a veces esta visión cuasi mecanicista es una aproximación útil, no es una buena representación ni siquiera de la física actual. La investigación en física moderna se ocupa de sistemas complejos, comportamientos emergentes, vastas simulaciones y resultados que son probabilísticos y estocásticos más allá de lo que implica la distribución gaussiana. Un ejemplo con paralelos en economía: variables termodinámicas agregadas como la temperatura no son suficientes para describir la rica dinámica de sistemas que están lejos del equilibrio; se requieren descripciones granulares de partículas individuales y sus distribuciones (Turrell et al., 2015a). Existen herramientas bien desarrolladas para abordar problemas que no son analíticamente manejables, que incluyen comportamientos complejos y presentan un alto grado de heterogeneidad. En la siguiente sección describimos una de ellas.

\section{EL MODELO DE MONTE CARLO}

En la década de 1930, el físico Enrico Fermi intentó resolver un problema muy difícil: el movimiento de uno de los tipos de partículas que componen el átomo, el neutrón, a través del material de fondo. Este era un cálculo complicado pues los neutrones tenían una distribución sobre varias variables, incluida la posición $r$, la energía $E$ y la dirección $\hat{\Omega}$ de acuerdo con una distribución $f(r, E, \hat{\Omega}, t) d r d E d \Omega$.

La ecuación completa rastrea la posición, dirección y evolución de la energía de $N$ partículas en el tiempo con términos que representan numerosas fuentes discretas y continuas e interacciones de fondo de los neutrones. Al inicio, Fermi y otros científicos intentaron resolver analíticamente todo el problema, con una sola ecuación, pero resultó difícil resolverla para todo los casos, excepto los más simples.

Fermi desarrolló un nuevo método para resolver estos problemas, con el que trató individualmente los neutrones, usando una sumadora mecánica para hacer los cálculos de cada neutrón a su vez. La técnica implicaba generar números aleatorios y compararlos con las proba- 
bilidades derivadas de la teoría. Si la probabilidad de que un neutrón colisionara era de 0,8 , y él generaba un número aleatorio menor que 0,8 , dejaba que un neutrón "simulado" colisionara. Se usaron técnicas similares para encontrar la dirección de salida del neutrón después de la colisión. Replicando esto para un gran número de neutrones simulados, Fermi pudo construir una imagen de la forma en que los neutrones pasarían a través de la materia. A Fermi le encantó asombrar a sus colegas con la exactitud de sus predicciones sin que, inicialmente, revelara su truco de tratar individualmente los neutrones (Metroplis, 1987).

E1 método más general del uso de números aleatorios para resolver problemas pronto recibió un nombre que reflejaba su carácter probabilístico: Monte Carlo. Fue desarrollado por Fermi, Stanislaw Ulam, John von Neumann y otros (Metropolis, 1987; Metropolis et al., 1953; Metropolis y Ulam, 1949). Tuvo amplio uso debido a la forma en que pondera naturalmente los escenarios que se exploran por la probabilidad de que ocurran. Es eficiente en problemas con alto número de dimensiones, y eficaz para reproducir todos los momentos de una función de distribución. La técnica de Monte Carlo es común en finanzas, donde se usa para calcular el valor esperado de los activos.

La simulación de Monte Carlo sigue siendo muy utilizada en diversas disciplinas, con nombres diferentes, entre ellos modelos basados en individuos en biología y ecología, modelos basados en agentes (MBA) en economía y sistemas multiagente en informática y logística, como se describe en Turrell (2016). Las aplicaciones recientes en física incluyen calcular cómo se pueden destruir células cancerígenas con haces de partículas (Arber et al., 2015; Bulanov y Khoroshkov, 2002), y cómo producir energía con reacciones de fusión nuclear (Lindl et al., 2004; Spears et al., 2015). Han dejado huella en ecología, donde se usan para modelar especies en peligro (Carter et al., 2015); en epidemiología, donde se usan para hacer predicciones detalladas de cómo se puede propagar la influenza dados los enlaces demográficos y de transporte (Degli Atti et al., 2008); y para el comportamiento descentralizado de vehículos autónomos (Ernest et al., 2016). Igual que en física, su uso en epidemiología consiste en remplazar un conjunto de ecuaciones diferenciales difíciles de resolver por simulaciones de agentes individuales. La simulación de Monte Carlo también pasa la prueba del mercado. Se ha usado para modelar la financiación de proyectos bajo incertidumbre, pronosticar tasas de amortización de hipotecas (Geanakoplos et al., 2012), rediseñar las reglas del índice NASDAQ(Bonabeau, 2002) y simular el transporte 
de personas (Heppenstall et al., 2011). Varias firmas, como Sandtable y Concentric, ofrecen modelos personalizados basados en agentes para aplicaciones comerciales.

\section{Gráfica 7}

Elementos de un modelo basado en agentes y sus interacciones

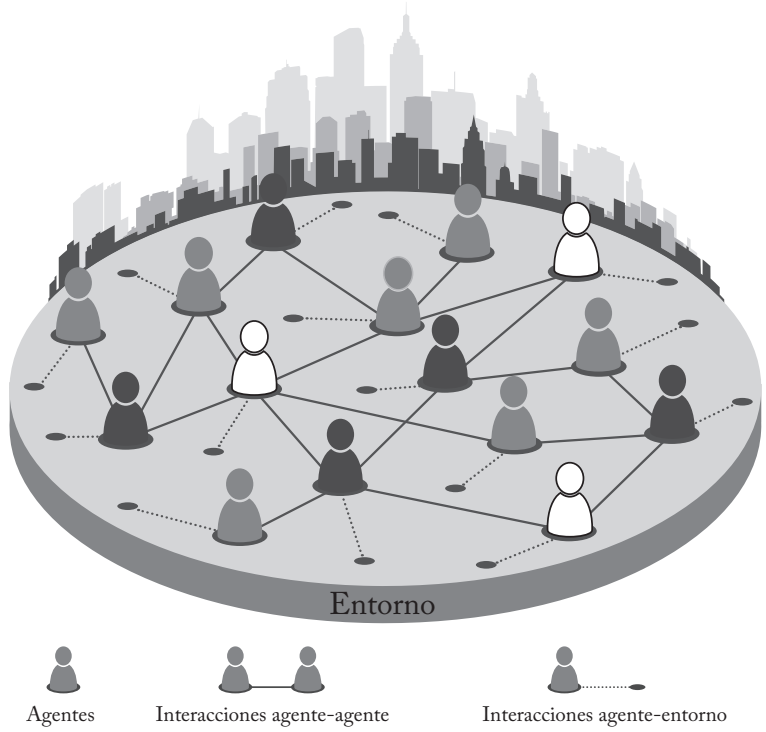

Fuente: Turrell (2016).

Los agentes de estos modelos pueden ser consumidores de una economía, peces de un cardumen e incluso galaxias del universo, como en Davis et al. (1985). Además de interactuar directamente entre sí, los agentes también pueden tener una conexión con su entorno; por ejemplo, bancos sujetos a regulación o ballenas que migran por el océano. Los comportamientos o reglas que siguen dependen de la pregunta de interés. Algunos modelos tienen muchos tipos de agentes, quizá firmas, trabajadores y gobiernos. Estos también pueden diferir, de modo que si bien todos los trabajadores tienen la posibilidad de ser empleados por una firma y recibir un salario, el capital humano y la propensión marginal a consumir de cada trabajador pueden ser diferentes y determinados de acuerdo con una distribución empírica.

La gráfica 7 muestra un esquema de un modelo económico basado en agentes. Los agentes heterogéneos interactúan entre sí en una estructura de red, y con el entorno más amplio.

La característica importante de los MBA es que explican la evolución general de un sistema simulando el comportamiento de 
cada agente individual y luego combinan explícitamente sus comportamientos de nivel micro para dar una imagen de nivel macro. Cada agente es una unidad autónoma que sigue un conjunto dado de reglas de comportamiento. Este enfoque de "abajo hacia arriba" se parece en espíritu al enfoque de "microfundamentos", aunque difiere fundamentalmente en cómo se agrega al nivel macro.

\section{¿EN QUÉ SON DIFERENTES LOS MODELOS BASADOS EN AGENTES EN ECONOMÍA?}

Existen importantes diferencias entre los MBA en ciencias y en economía. En economía, los comportamientos a nivel de agente no se conocen con el mismo nivel de exactitud que las leyes de la naturaleza que rigen las interacciones entre partículas, por ejemplo. En economía, los comportamientos pueden cambiar en el tiempo en respuesta al entorno. Por tanto, en economía los supuestos a nivel de agente se deben probar y variar rigurosamente.

En parte como consecuencia de la incertidumbre inherente al comportamiento de los agentes, los MBA en economía solo pueden ajustar los datos probabilísticamente. Tienden a ajustar momentos y reproducir hechos estilizados. Algunos argumentan que esto es ingenuo en comparación con el ajuste más exacto de la evolución histórica de variables en el tiempo. Pero este último enfoque, que a menudo usa procesos de forzamiento, parece sugerir un nivel de precisión inverosímil. Un MBA es una manera de generar muchas realizaciones posibles y plausibles de las variables, del mismo modo que se generan diferentes trayectorias posibles de precios con la fijación de precios de opciones de Monte Carlo. Otra manera de pensar esto es en términos del trade-offentre sesgo y varianza. En general, los MBA obtienen un sesgo menor al costo de una varianza mayor. Los errores del modelo son cuadráticos en el sesgo pero solo son lineales en la varianza (Friedman et al., 2001). Los modelos con procesos de forzamiento apuntan a una varianza baja al costo de un sesgo mayor.

¿Dónde encajan los MBA en el panorama de modelación más amplio? La gráfica 8 lo muestra esquemáticamente. Los modelos se sitúan en un espectro. Los modelos estadísticos rara vez dicen algo sobre agentes heterogéneos. Los modelos DSGE dicen más y los MBA aún más. Pero los MBA no son útiles para todos los problemas. Aunque en principio se podrían usar para pronosticar, ya existen modelos potencialmente mejor equipados para ello, como los modelos de factores dinámicos (Stock y Watson, 2011) y de aprendizaje 
automático (Chakraborty y Joseph, 2017). Los MBA están mejor situados para hacer pronósticos condicionales, donde se explora una política particular. Así es como los epidemiólogos usan los modelos basados en agentes (Degli Atti et al., 2008): en vez de intentar predecir el momento específico en que ocurrirá un brote de virus, identifican los factores de riesgo para que un virus estalle y luego se difunda. En la gráfica 8, los MBA anidan los modelos DSGE como un caso especial con poca heterogeneidad, sin variables de nivel y con un conjunto particular de supuestos sobre el comportamiento de los agentes. Definidos en forma amplia, los MBA agregarán más valor cuando los problemas giren en torno a heterogeneidad, complejidad, no linealidad, emergencia, heurísticas y reglas detalladas.

\section{Gráfica 8}

Consistencia y tipos de agentes en diversos modelos

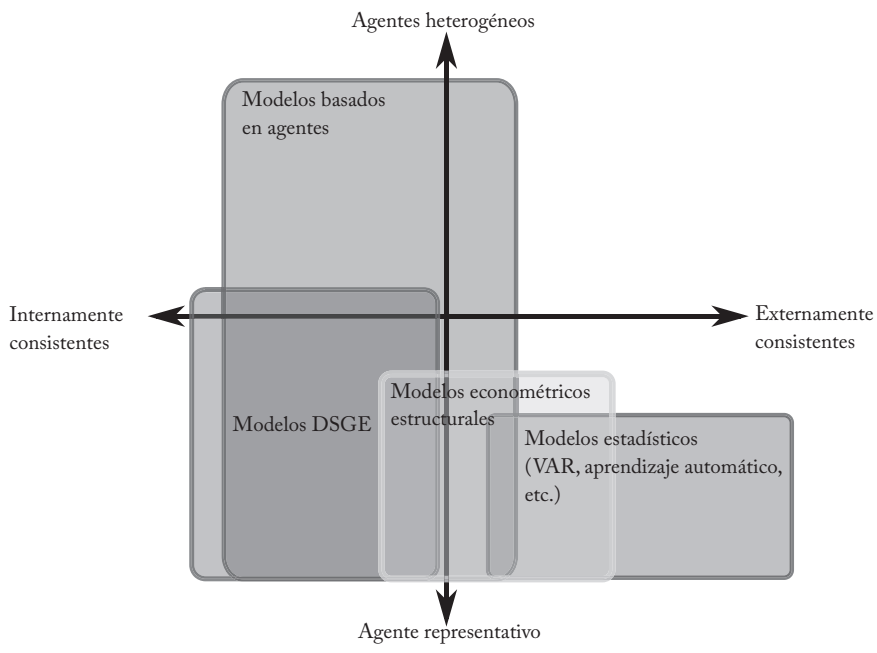

Se puede pensar que los MBA macroeconómicos se sitúan en un espacio de modelación más amplio, que aquí se muestra con dos ejes. La consistencia interna se representa mejor mediante un comportamiento fuertemente microfundamentado, mientras que la consistencia externa se demuestra mediante la concordancia con los datos. En el otro eje está el grado de heterogeneidad de los agentes que puede incluir el modelo, con agentes representativos en un extremo y heterogeneidad en muchas dimensiones en el otro. Es posible que la variación dentro de cada tipo de modelo sea mayor que la variación entre ellos, pero la gráfica ilustra su ubicación aproximada en el espacio de modelación más amplio.

Los modelos de la gráfica 8 requieren enfoques bastante diferentes y los MBA tienen una filosofía de modelación distinta de sus vecinos más cercanos. E1 modelo DSGE arquetípico está atado a una serie de supuestos, incluidas expectativas racionales. En vez de ofrecer un modelo "básico", los MBA son un conjunto flexible de herramientas para resolver problemas complejos que involucran agentes heterogéneos. Se podrían usar expectativas racionales, pero no hay un requisito 
para hacerlo. Esta flexibilidad es una razón para que los MBA pueblen todos los campos, desde juegos de guerra hasta ecología, y por qué es imposible escribir un MBA representativo.

Como demostración, el cuadro 2 muestra una lista no exhaustiva de funciones de consumo en diferentes MBA macroeconómicos. En los modelos DSGE ha surgido un modelo básico, en parte porque la necesidad de manejo analítico obliga al modelador a elegir entre un conjunto restringido de comportamientos. Los MBA son una generalización para más comportamientos y más agentes. Esto significa que suelen ser personalizados, adaptados a la pregunta particular que responden. Por esa razón, han sido criticados como "caja negra”. $\mathrm{Su}$ carácter personalizado significa que el costo de entenderlos es también mayor.

Pero no hay ninguna razón para que un MBA no pueda tener lo que Blanchard (2017) considera requisito de un modelo macroeconómico básico: rigideces nominales, racionalidad limitada y horizontes limitados, mercados incompletos y un papel de la deuda. De hecho, estas características se encuentran en muchos MBA macroeconómicos. Una de las principales diferencias es el grado en que estos modelos se resuelven en forma computacional. El proceso de un modelo DSGE típico es especificar los comportamientos de los agentes y agregarlos analíticamente, suponiendo que los mercados se despejan. Usualmente se linealizan y en el último paso se resuelven numéricamente. En un MBA típico, el proceso es bastante diferente. Se resuelven numéricamente a nivel de agentes, un comportamiento a la vez.

Esto tiene varias implicaciones para las fortalezas y debilidades de los MBA. Estar libres de la necesidad de especificar ecuaciones que puedan encajar y resolverse analíticamente puede ser liberador para algunos problemas. Esto no significa que no se puedan representar matemáticamente: hay un teorema que dice que todos los MBA que se pueden calcular numéricamente tienen una representación matemática explícita (Epstein, 2006; Leombruni y Richiardi, 2005) ${ }^{1}$.

Pero la traducción matemática directa suele ser muy difícil de transcribir y solo es interesante en casos especiales. Es mejor pensar en los MBA como algoritmos para agregar comportamientos de actores individuales que como sistemas de ecuaciones.

Si esta flexibilidad tiene una desventaja es que se debe remplazar la certeza analítica por la convergencia numérica. Sinitskaya y Tesfat-

1 Todo modelo basado en agentes es computable por una máquina de $\mathrm{Tu}$ ring, y todo algoritmo computable por una máquina de Turing se puede expresar mediante conjuntos de funciones recursivas parciales. 
sion (2015) destacan esto comparando soluciones numéricas con un problema de optimización del ciclo de vida dentro de un MBA con una solución analítica conocida. Los métodos de solución numérica no logran exactamente el mismo resultado que la solución analítica. Pero para la mayoría de los problemas en macroeconomía, es posible que la precisión en la forma de plantear un problema sea una fuente de error mucho mayor que la falta de precisión en la solución numérica de ese problema. De hecho, este es otro ejemplo del trade-off sesgo-varianza.

\section{Cuadro 2}

Ejemplos de consumo usados en diferentes modelos MBA macroeconómicos

\begin{tabular}{|c|c|c|}
\hline Descripción del modelo de consumo & $\begin{array}{l}\text { Referencias del modelo } \\
\text { de consumo }\end{array}$ & $\begin{array}{l}\text { Referencias del } \\
\text { MBA }\end{array}$ \\
\hline $\begin{array}{l}\text { Fracción dependiente de la inflación del } \\
\text { ingreso permanente; consumo dado por } \\
c_{t}=k_{t} \hat{y} t \text { donde } k_{t}=k_{t-1}-f\left(i_{t}-\mathbb{E} \pi_{t+1}-r_{t}\right)\end{array}$ & $\begin{array}{l}\hat{y} t \text { es el ingreso per- } \\
\text { manente de Friedman } \\
(1957) .\end{array}$ & Salle et al. (2013) \\
\hline $\begin{array}{l}\text { Consumo agregado como suma del ingresos } \\
\text { de todos los empleados y desempleados; } \\
C_{t}=\sum_{u, c} \sum_{i} y_{i, t}\end{array}$ & $\begin{array}{l}\text { Consumidores de boca } \\
\text { a boca descritos en } \\
\text { Campbell y Mankiw } \\
(1989) \text {. }\end{array}$ & Dosi et al. (2010) \\
\hline $\begin{array}{l}\text { Propensiones fijas } \alpha_{1} y \alpha_{2} \text { del ingreso real } \\
\text { disponible esperado y de la riqueza real espe- } \\
\text { rada; } C_{t}=\left[1 / \mathbb{E} p_{t+1}\right]\left(\alpha_{1} y_{t}+\alpha_{2} W_{t}\right)\end{array}$ & $\begin{array}{l}\text { Godley y Lavoie } \\
(2007)\end{array}$ & Caiani et al. (2016) \\
\hline $\begin{array}{l}\text { Expectativa adaptativa (con parámetro de } \\
\text { memoria } \xi \text { ) del ingreso y fracción fija de } \\
\text { riqueza (basada en reservas de seguridad); } \\
c_{t}=\xi_{t-1}+(1-\xi) y_{t}+0,05 W_{t}\end{array}$ & Carroll $(1997,2009)$ & Assenza et al. (2015) \\
\hline $\begin{array}{l}\text { Aumento cóncavo monotónicamente limita- } \\
\text { do por encima de la fracción de riqueza real; } \\
c_{t}=\min \left[\left(y_{t} / p_{t}\right)^{\alpha}, y_{t} / p_{t}\right] \\
\operatorname{con} 0<\alpha<1\end{array}$ & $\begin{array}{l}\text { Carroll y Kimball } \\
\text { (1996); Souleles (1999) }\end{array}$ & Lengnick (2013) \\
\hline $\begin{array}{l}\text { Crecimiento de la riqueza } \Delta W \text {, consumo his- } \\
\text { tórico promedio de otros hogares } \bar{c}_{t} \text { y un nivel } \\
\text { de consumo de referencia } \tilde{c} t \\
c_{t}=\tilde{c t}+\alpha\left(\Delta W / p_{t-1}\right)+\left(\bar{c}_{t-1}-c_{t-1}\right)\end{array}$ & $\begin{array}{l}\text { Abel (1990); Jawadi y } \\
\text { Sousa (2014) }\end{array}$ & Guerini et al. (2016) \\
\hline $\begin{array}{l}\text { Maximización de la utilidad resuelta numé- } \\
\text { ricamente sujeta a restricción presupuestal } \\
\text { intertemporal con ocio }(1-l) \text { y riqueza } W \\
\max _{c, l} \otimes\left\{\sum_{r=t}^{\infty} \beta^{r-t} u\left(c_{t}, 1-l_{t}\right)\right\} \\
\text { tal que } W_{t-1}-p_{t} c_{t}>0, c_{t}>0\end{array}$ & Friedman (1957) & $\begin{array}{l}\text { Sinitskaya y Tesfat- } \\
\text { sion }(2015)\end{array}$ \\
\hline $\begin{array}{l}\text { Consumo como fracción fija de la riqueza y el } \\
\text { ingreso; } c_{t}=\alpha\left(W t+y_{t}\right)\end{array}$ & $\begin{array}{l}\text { Godley y Lavoie } \\
(2007)\end{array}$ & $\begin{array}{l}\text { Gatti y Desiderio } \\
(2015) ; \text { Gualdi et al. } \\
(2015)\end{array}$ \\
\hline $\begin{array}{l}\text { Reserva de seguridad con ingreso promedio } \\
\text { retrasado } \tilde{y} t \text { y meta de relación riqueza a } \\
\text { ingreso } \phi ; c_{t}=\tilde{y} t+\alpha\left(W t-\phi y_{t}\right)\end{array}$ & Carroll (1997) & $\begin{array}{l}\text { Chan y Steiglitz } \\
\text { (2008); Cincotti et } \\
\text { al. (2010); Dawid et } \\
\text { al. }(2012,2014)\end{array}$ \\
\hline
\end{tabular}

Para todos los detalles, ver las referencias. 
La filosofía de modelación también difiere en la interpretación de los resultados. Es útil pensar en los MBA como una máquina para generar muchas realizaciones alternas del mundo. Igual que en los experimentos reales, es útil hacer muchas repeticiones con grupos de control y de tratamiento. Si la distribución futura de una variable particular fuese realmente incierta, digamos un ingreso $y$ tal que $y \sim f$, se podría correr un MBA no solo con millones de extractos posibles de la misma distribución $f$ sino también de una distribución paramétrica o empírica totalmente diferente $g$. En un MBA, es posible hacerlo modificando una sola línea de código. En modelos más analíticos, podría no ser posible en absoluto. Debido a la complejidad de las interacciones dentro de un MBA, también puede ser necesario tratar los resultados como si fueran datos experimentales reales, con la maquinaria de verificación de hipótesis e intervalos de confianza.

Como sucede con todos los modelos, carece de sentido tener más parámetros libres que parámetros impuestos o calibrados. Se ha acusado a los MBA de estar llenos de parámetros libres, pero esta es una mala modelación y no un rasgo intrínseco de los MBA. Una medida de todos los modelos es con cuánta parsimonia describen los datos empíricos. Los MBA macroeconómicos pueden ajustar hechos estilizados micro y macro para la mayoría de las entradas del cuadro 1, si no para todas. Por ejemplo, Gualdi et al. (2015) y Caiani et al. (2016) ajustan la frecuencia y el grado de ciclicidad de la productividad, los salarios nominales, la deuda de las empresas, las ganancias de los bancos, la inflación, el desempleo, los precios y las pérdidas crediticias, mientras que Dosi et al. (2015) reproducen las distribuciones del crecimiento del producto y la duración de las crisis bancarias, entre otras características. Al simular cada actor individual de un sistema económico, todos los momentos de las funciones de distribución son accesibles para los MBA.

$\mathrm{Si}$, como se describe en Wren (2016b), los modelos DSGE mantienen la consistencia interna sacrificando alguna consistencia externa, son más un puente entre consistencia interna y externa: sacrifican cierta consistencia interna permitiendo que los agentes tengan comportamientos que no son híper racionales. No obstante, la combinación de modelos personalizados, flexibilidad y la tendencia a centrarse en sistemas complejos puede significar que la comunicación es un reto para los modeladores basados en agentes. Los MBA rara vez son tan fáciles de escribir en forma de ecuación como, por ejemplo, el modelo DSGE nuevo keynesiano simple de tres ecuaciones. Quizá no siempre haya una solución fácil: los sis- 
temas complejos reales a veces se deben describir con simulaciones complejas, al menos inicialmente.

Durante mucho tiempo la ciencia ha tenido que abordar el trade-off entre modelos más grandes, con más características, y modelos más abstractos pero más fáciles de entender. Han surgido compromisos. En física, es común usar una simulación compleja de un sistema como medio inicial para explorar hipótesis o descubrir nuevos fenómenos. Una vez se identifica un efecto específico en la simulación compleja, se construye un modelo puramente teórico (o un modelo numérico mucho más simple) para explicar sus principales características. Un ejemplo se encuentra en Turrell et al. (2015b), en el que se identificó por primera vez una manera muy rápida para que los láseres calentaran materia con un modelo de más de $10^{7}$ agentes y luego se explicó con pocos parámetros, en un modelo analítico que usaba ecuaciones diferenciales. Otro ejemplo de un modelo teórico derribado por simulación se encuentra en Sherlock et al. (2014). El mal desempeño de la teoría original, aceptada durante décadas, habría sido difícil de entender o describir analíticamente sin utilizar un MBA.

Gualdi et al. (2015) son ejemplos de este enfoque en un MBA macroeconómico. Un modelo rico y complejo se reduce a un MBA mucho más simple que mantiene, y explica, el mismo fenómeno. La diferencia entre este enfoque y empezar simplemente con un modelo más pequeño, quizá analítico, es que el modelo más grande y más realista sorprende al investigador con una relación o un fenómeno que no esperaba. La tarea del investigador es entonces identificar este efecto e interrogarlo aún más.

En suma, que un MBA sea bueno o malo depende de sus supuestos específicos, de cómo se usa y cómo se interpretan los resultados. La falta de restricciones a los supuestos de modelación puede ser un peligro. Como lo que genera este peligro es la flexibilidad de los MBA, los beneficios de esta flexibilidad deben ser significativos para justificar este costo. En la siguiente sección se argumenta que lo son.

\section{¿QUÉ PUEDEN LOS MBA HACER POR LA ECONOMÍA?}

La fortaleza de los MBA para la economía radica en su flexibilidad con respecto a los demás modelos de la gráfica 8. Fabio Ghironi identificó temas que quizá sean importantes para los modelos macroeconómicos en el futuro. Son temas que pueden ser difíciles de captar en los modelos establecidos. Pero, como se muestra en el cuadro 3, los MBA ya ofrecen nuevas perspectivas sobre cada uno de ellos. 
En general, los MBA son adecuados para situaciones donde son importantes las interacciones entre agentes, donde predominan la heurísticas, donde es importante la heterogeneidad de agentes, donde las políticas tienen implicaciones a nivel de agentes, donde son abundantes los datos granulares y donde fallan los métodos analíticos. Las distribuciones no gaussianas, las ecuaciones no lineales, las elecciones inconsistentes en el tiempo, los comportamientos limitadamente racionales; todo ello se puede resolver numéricamente a nivel de agentes. Puesto que muchos problemas del mundo real involucran estas características, los MBA tienen muchos usos potenciales en economía (Tesfatsion, 2002).

Cuadro 3

Temas que se consideran importantes para el futuro de la macroeconomía y los MBA que los estudian

\begin{tabular}{lll}
\hline Tema & Referencia del MBA & Descripción del MBA \\
\hline $\begin{array}{l}\text { Intermediación financiera } \\
\text { heterogeneidad de firmas y }\end{array}$ & Ashraf et al. (2017) & $\begin{array}{l}\text { Análisis del papel de los } \\
\text { bancos en la entrada y salida } \\
\text { de firmas. }\end{array}$ \\
\hline Granularidad y redes & Bardoscia et al. (2017) & $\begin{array}{l}\text { Explora cómo se vuelven } \\
\text { heterogéneas en productivi- } \\
\text { dad las firmas exportadoras y } \\
\text { locales. }\end{array}$ \\
\hline & $\begin{array}{l}\text { Muestra que la diversificación } \\
\text { puede llevar a un aumento } \\
\text { del riesgo sistémico en redes } \\
\text { bancarias. }\end{array}$ \\
\hline $\begin{array}{l}\text { Interdependencia de políticas } \\
\text { Modelo macroeconómico que }\end{array}$ & $\begin{array}{l}\text { Popoyan et al. (2016) } \\
\text { explora la interdependencia } \\
\text { entre regulación macropru- } \\
\text { dencial y política monetaria. }\end{array}$ \\
\hline
\end{tabular}

Gran parte de la investigación en redes, en particular la que se refiere a sistemas financieros, ha demostrado que a nivel de sistema existen propiedades emergentes que surgen de las interacciones a nivel de agentes (Battiston et al., 2007; Gai et al., 2011). Por ejemplo, Bardoscia et al. (2017) muestran que a medida que los bancos se integran y diversifican a nivel de agentes, pueden aumentar el riesgo de inestabilidad sistémica porque crean dependencias cíclicas que amplifican la tensión. Los MBA pueden captar este comportamiento donde las interacciones a nivel de agentes llevan a un comportamiento contraintuitivo a nivel macro. Los efectos de rebaño son otro ejemplo (Alfarano et al., 2005).

Uno de los usos más importantes de los MBA es la exploración de microfundamentos diferentes de expectativas racionales. El cuadro 2 muestra cómo manejan los MBA la gama total de supuestos de com- 
portamiento de consumo. Hay un conjunto emergente de evidencia experimental que sugiere que las heurísticas pueden funcionar mejor que los comportamientos "racionales"(Gigerenzer y Brighton, 2009). Para tener confianza en las conclusiones de los MBA, es necesaria una serie más amplia de trabajos sobre la mejor manera de incluir comportamientos realistas a nivel de agentes; para un ejemplo, ver Gabaix (2016). Y es probable que para entender los comportamientos de los agentes, las técnicas avanzadas de aprendizaje automático sean útiles para aproximar las decisiones que toman personas reales. DeepMind ya ha dado pasos iniciales en este camino (Leibo et al., 2017).

Los MBA quizá se distinguen más fácilmente de otros modelos por su capacidad para modelar la heterogeneidad (Hommes, 2006). Hay creciente evidencia de la importancia de la heterogeneidad de hogares, empresas y consumidores tanto en estatus (como la riqueza) como en comportamiento (Alfi et al., 2009; Gabaix, 2011; Guvenen, 2011). Un ejemplo especial es cuando importan las variables de nivel de los agentes. Bajo los supuestos del modelo DSGE arquetípico no importa rastrear variables como la riqueza o la deuda porque los flujos describen implícitamente la información de los niveles relevantes para el modelo. Pero hay situaciones en las que es relevante información más detallada de los niveles, como cuando agentes heterogéneos buscan un nivel particular de riqueza o de deuda (Elmendorf et al., 1996; Muellbauer y Murata, 2009) o cuando la riqueza retroalimenta el comportamiento (Cooper y Dynan, 2016).

Desde hace algún tiempo existen modelos que incluyen identidades contables a nivel agregado (Wren, 2016a). Pero el reciente MBA de Caiani et al. (2016) los aplica a nivel de agentes para captar la interacción de las decisiones de los agentes a través del canal del balance. Gatti y Desiderio (2015) muestran el papel de los balances de las empresas en política monetaria. También se deben modelar las políticas que actúan de manera heterogénea; por ejemplo, la política del Comité de Política Financiera del Banco de Inglaterra en 2015 para garantizar "que los prestamistas hipotecarios no extiendan más del $15 \%$ del número total de nuevas hipotecas residenciales a relaciones de préstamos a ingresos iguales o superiores a 4,5”. Para la política monetaria, uno de los canales prácticos importantes para influir en el consumo depende de su efectividad sobre la heterogeneidad de los agentes: los que se benefician de la política de flexibilización tienen mayores propensiones marginales a consumir que los que pierden (Auclert, 2015). La presidenta de la Reserva Federal de Estados Unidos, Janet Yellen, señaló: 
La comprensión de los "economistas" de cómo afectan la economía los cambios de política fiscal y monetaria también se podría beneficiar si se reconoce que los hogares y las firmas son heterogéneos. Por ejemplo, en los modelos simples del mecanismo de transmisión monetaria de libro de texto, los bancos centrales operan en gran medida a través del efecto de las tasas de interés reales sobre el consumo y la inversión. Una vez se tiene en cuenta la heterogeneidad, surgen otros canales importantes. Por ejemplo, el gasto de muchos hogares y firmas parece ser muy sensible a los cambios en el ingreso laboral, en las ventas de las empresas o en el valor de las garantías, que a su vez afectan su acceso al crédito; condiciones que la política monetaria solo afecta indirectamente. Estudiar más de cerca modelos monetarios con agentes heterogéneos podría ayudar a arrojar nueva luz sobre estos aspectos de los mecanismos de transmisión monetaria (Yellen et al., 2016).

Se podría usar un MBA para ver de qué manera la heterogeneidad en varias dimensiones interrelacionadas afecta la transmisión de políticas; por ejemplo, la propensión marginal a consumir como función de los activos netos y la demografía. La desigualdad es un tipo de heterogeneidad que ya se explora. Para ejemplos donde distribuciones inicialmente igualitarias, como el ingreso, se vuelven desiguales endógenamente, ver Gibson (2007) y Caiani et al. (2016). Los MBA no son la única manera de incluir heterogeneidad, pero ofrecen un camino más fácil hacia un mayor grado de heterogeneidad. Así como Fermi y sus colegas encontraron en el caso de los neutrones, hay un punto donde el trade-off entre modelos matemáticos cada vez más elaborados y modelos resueltos numéricamente favorece a estos últimos. Los nuevos tipos de modelos DSGE están más cerca de este umbral, pero el otro lado del trade-off sigue siendo digno de atención.

La capacidad para modelar sistemas sin restricciones analíticas facilita el estudio de algunos problemas. Lord Stern, autor de una excelente revisión de la economía del cambio climático (Stern, 2007), señala que la modelación basada en agentes es una forma más realista de incorporar los trade-offs macroeconómicos del cambio climático (Stern, 2016b). Para un ejemplo de un MBA macroeconómico que afronta este desafío, ver Lamperti et al. (2017a). Esta capacidad también facilita el análisis de sistemas que están fuera del equilibrio, sistemas donde los mercados no necesariamente se despejan, que están en desequilibrio dinámico o en transición entre diferentes equilibrios. Una de las perspectivas únicas de este tipo de modelos es que muestran cómo puede surgir un desequilibrio como resultado de elecciones de agentes individuales que buscan el interés personal (pero no necesariamente híper racionales).

Un ejemplo dramático es el MBA macroeconómico dinámico no lineal de Gualdi et al. (2015), en el que el producto y el empleo 
colapsan en forma discontinua a nuevos valores dependiendo de la propensión de las firmas a contratar y despedir nuevo personal y de sus niveles de deuda. Estos pasos discontinuos de un equilibrio a otro son bien conocidos en física como "transiciones de fases". También se han identificado en el contagio de redes financieras (Gai y Kapadia, 2010; Watts, 2002) y en la forma en que cambian las opiniones y narrativas dentro de una población (Shiller, 2017; Sornette, 2014). De Grauwe (2010) desarrolla un MBA que incluye este efecto de cambio de opinión en un esquema nuevo keynesiano.

Es indudable que también hay problemas. La calibración, por ejemplo, es un área relativamente débil de los MBA macroeconómicos. Aunque los mejores han logrado reproducir una impresionante gama de hechos estilizados, las técnicas y estándares de calibración tienden a variar sustancialmente entre modelos. El método generalizado de momentos es un enfoque comúnmente utilizado (Franke y Westerhoff, 2012), pero también se están desarrollando técnicas basadas en autorregresiones de vectores y aprendizaje automático (Guerini y Moneta, 2017; Lamperti et al., 2017b).

Los MBA ya han dado sólidos resultados sobre algunos sistemas económicos y financieros parciales (no generales). Dan explicaciones plausibles de fenómenos de los mercados financieros, incluidas las colas gruesas, la volatilidad agrupada y las burbujas (Alfi et al., 2009; Cutler et al., 1989; Hong y Stein, 1999; Lux y Marchesi, 1999). Lux y Marchesi demostraron que para reproducir las colas gruesas observadas en la distribución de los rendimientos absolutos de los mercados se requiere que un número de participantes negocie no con base en los fundamentos sino con base en el optimismo y el pesimismo (conocidos como corredores de ruido). En su modelo, los agentes pueden cambiar de grupo con base en el desempeño de las estrategias. Los fundamentos del mercado siguen una distribución gaussiana, de modo que la distribución de cola gruesa de los rendimientos solo se debe a las interacciones entre los diferentes tipos de corredores. Si los agentes ven la oportunidad de obtener ganancias convirtiéndose en corredores de ruido, cambian de estrategia. En el corto plazo, pueden montarse en una oleada de optimismo o de pesimismo, y así obtener mayores rendimientos absolutos de los que implicaría la variación de los fundamentos. Pero la desviación del valor subyacente tiene límites debido a los agentes fundamentalistas restantes y, eventualmente, los precios deben revertir parcialmente. Este modelo simple basado en agentes da una explicación convincente de uno de los enigmas de los mercados financieros. 
Gráfica 9

Esquema del mercado de bonos corporativos
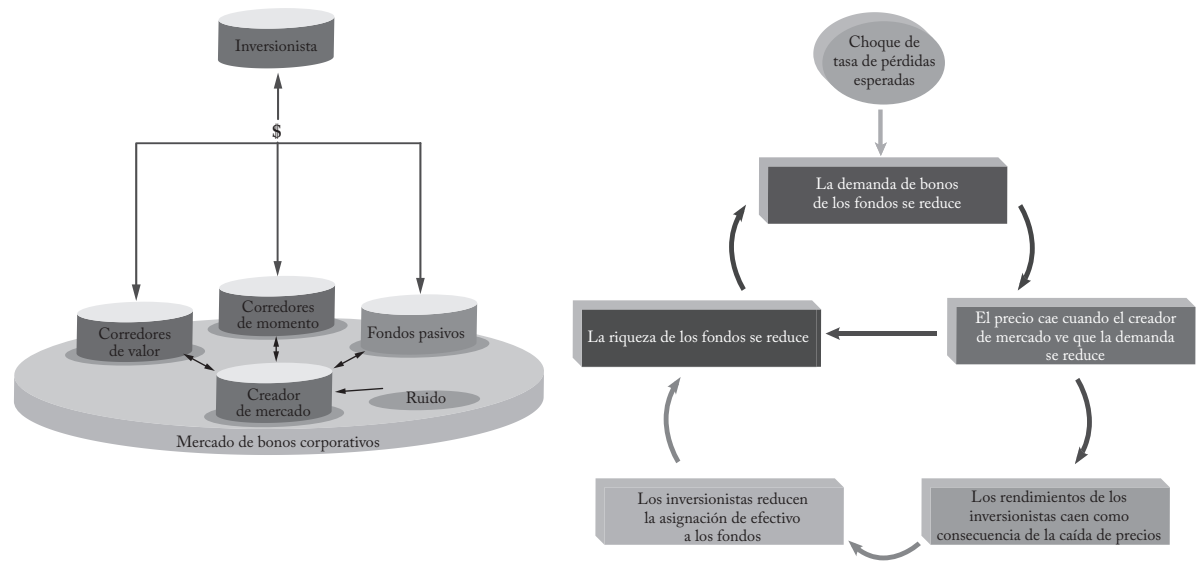

(a) Esquema del MBA de negociación

en el mercado de bonos corporativos

Fuente: Braun-Munzinger et al. (2016).

En el Banco de Inglaterra, los MBA se han usado para ayudar a entender el mercado de bonos corporativos (Braun et al., 2016) y el mercado de vivienda del Reino Unido (Baptista et al., 2016). E1 modelo anterior, que se muestra esquemáticamente en la gráfica 9a, se usó para estudiar de qué manera los inversionistas que canjean los bonos corporativos que mantienen por fondos de inversión abiertos pueden generar bucles de realimentación en los que caen los precios de los bonos. El bucle no lineal induce disparadas de precios del índice de los bonos (que se muestran esquemáticamente en la gráfica 9b) y el modelo analiza posibles maneras de reducir la magnitud de las disparadas. El modelo está calibrado con datos empíricos granulares y produce un ajuste razonable con la distribución de los rendimientos diarios del logaritmo de precios. En un escenario aumenta la fracción de fondos que usan estrategias de negociación pasivas.

La mayor presencia de fondos pasivos estabiliza el precio y el rendimiento en el caso mediano, pero introduce un riesgo de cola de caídas muy grandes de precios (aumentos de rendimiento), como se muestra en la gráfica 10 . La razón es que cuando que hay pocos fondos que comercian activamente, es más probable que el creador del mercado observe un exceso súbito de demanda neta positiva o negativa y, en respuesta, genere importantes movimientos de precios. Este no es un resultado que se esperaría a priori. 
Gráfica 10

Rendimiento mediano del índice de un bono corporativo

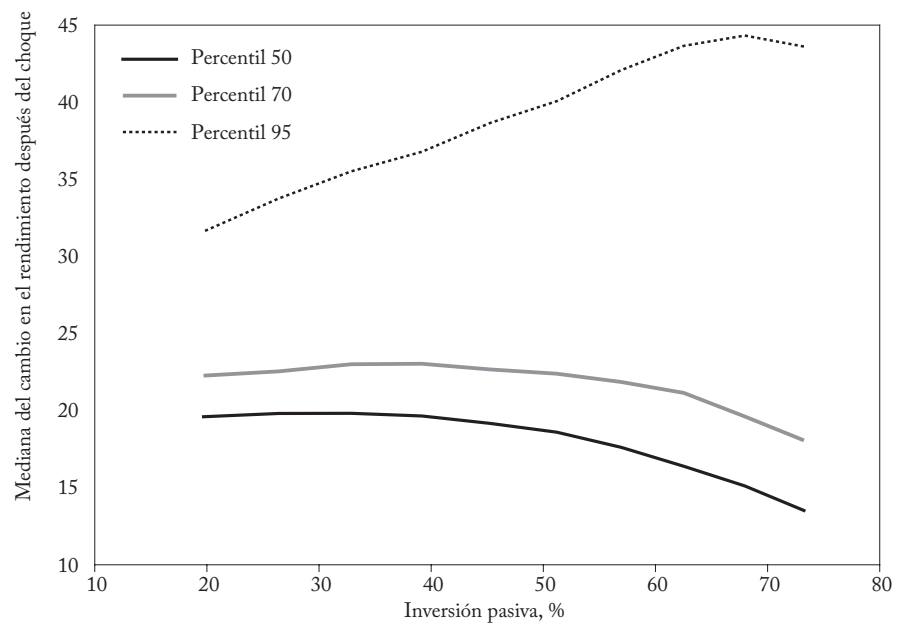

Distribución de los resultados del rendimiento mediano del índice de un bono corporativo 100 días de negociación después de un aumento súbito de la tasa de pérdidas sobre el índice. Los percentiles se refieren a corridas repetidas del modelo.

Fuente: Braun-Munzinger et al. (2016).

Un modelo del mercado de vivienda del Reino Unido desarrollado en el Banco de Inglaterra explora los vínculos entre estabilidad macroeconómica y ciclos del precio de la vivienda (Erlingsson et al., 2014; Geanakoplos et al., 2012). No es sencillo captar estas dinámicas cíclicas. Una de las razones posibles es que el mercado de vivienda incluye muchos tipos de agentes: arrendatarios, compradores por vez primera, propietarios ocupantes, vendedores y propietarios arrendadores. Todos ellos se representan en el MBA, y son heterogéneos por edad, balance bancario, ingreso, endeudamiento y ubicación. Además hay un sector bancario (un prestamista hipotecario) y un banco central; todos se muestran esquemáticamente en la gráfica 11a. La combinación de las acciones de estos agentes genera la dinámica cíclica que muestra la gráfica $11 \mathrm{~b}$, la cual surge endógenamente en el modelo.

La inclusión explícita de un sector bancario es importante porque los bancos dan crédito hipotecario a los hogares y fijan los términos y condiciones a los prestatarios. Las decisiones de préstamo del sector bancario están sujetas a la regulación del banco central, que establece las políticas de relaciones préstamo a ingreso $(\mathrm{P} / \mathrm{I})$, préstamo a valor $(\mathrm{P} / \mathrm{V})$ y cobertura de intereses $(\mathrm{CI})$. E1 modelo reproduce la función de densidad de la proporción de préstamos por franja de préstamo a ingreso tomada de la base de datos de ventas de productos hipotecarios del Reino Unido, como se muestra en la gráfica 12. E1 modelo se ha 
usado para analizar diversos escenarios, incluso escenarios de política. Por ejemplo, una política que se aplica a nivel de agentes -como no más del 15\% de nuevas hipotecas para relaciones préstamo-ingreso iguales o mayores que un múltiplo dado- sirve para atenuar los ciclos de auge y caída de los precios de la vivienda a nivel agregado.

\section{Gráfica 11}

Modelo del mercado de vivienda
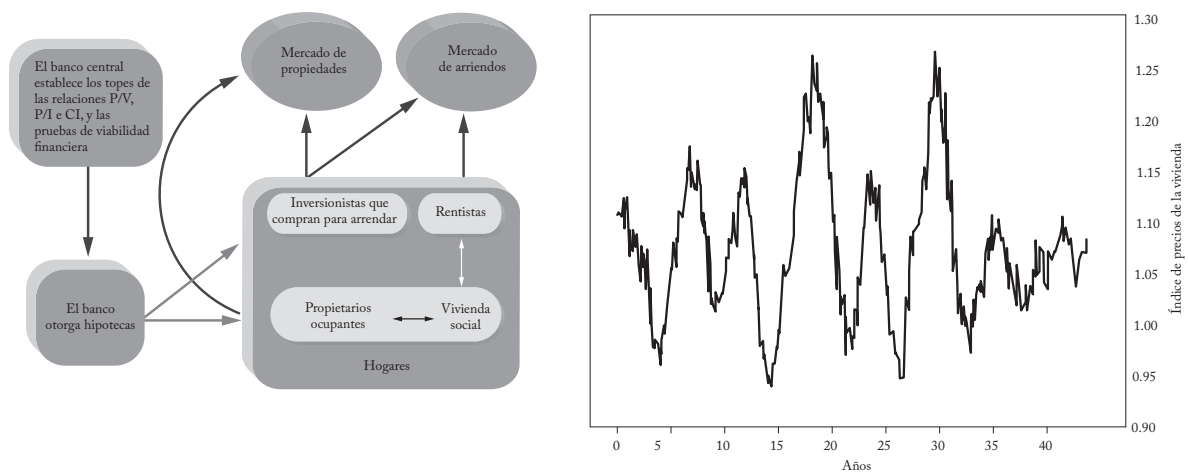

(a) Esquema de los agentes y las interacciones en el modelo del mercado de vivienda.

(b) Corrida de referencia del modelo del mercado de Fuente: Baptista et al. (2016). vivienda que muestra ciclos de su índice de precios.

\section{Gráfica 12}

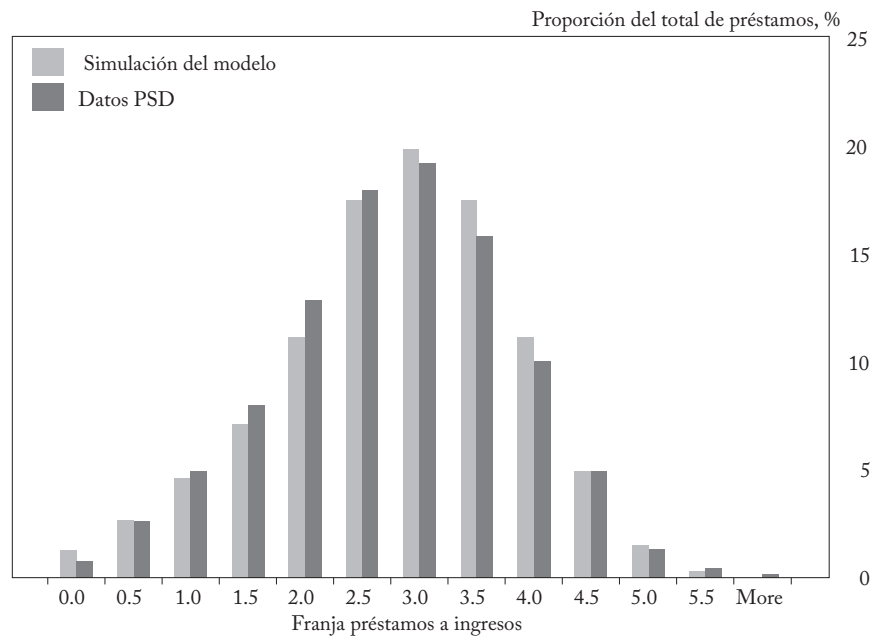

Una vez calibrado, el MBA del mercado de vivienda reproduce la función de densidad de la relación préstamos a ingresos en el Reino Unido.

Fuente: Baptista et al. (2016). 


\section{CONCLUSIÓN}

La economía ha sido inusualmente insular y hay poca confianza en los economistas. En el centro de esta insularidad ha estado un tipo particular de comportamiento microfundamentado. Pero el tipo de microfundamentación incorporado en los modelos macroeconómicos convencionales está lejos de ser la única opción o, en algunos casos, la más plausible. Y los modelos microfundamentados no son el único tipo de modelos útiles para dar sentido a las fluctuaciones económicas agregadas. Un enfoque más diverso de la modelación macroeconómica puede ser beneficioso para dar sentido a la economía y establecer políticas que den forma a la economía.

Con base en las últimas siete décadas de simulación, este escrito presenta un enfoque de modelación complementario. En los MBA, el comportamiento agregado se basa en el comportamiento de los agentes individuales. En ese sentido, comparte el espíritu de la "revolución de los microfundamentos", aunque la filosofía de modelación es diferente. Estos enfoques ya han mostrado su valía en entornos de (des)equilibrio parcial, en finanzas y en ciencias naturales.

Pero hay trabajo por hacer antes de que los MBA disfruten de la misma posición que sus primos de la literatura económica convencional. Entre los temas más fundamentales que se deben abordar están: 1. ¿cuáles son los comportamientos más realistas que se deben incorporar, a nivel de agentes, una vez se relaja el supuesto de agentes representativos con expectativas racionales?

2. ¿cómo captar mejor el flujo de información entre agentes dentro de la macroeconomía?, y

3. ¿en qué situación es probable que los modelos MBA y DSGE den resultados similares/diferentes?

Además de estas preguntas técnicas, los modeladores también se deben preguntar cómo se pueden comunicar mejor los resultados de simulaciones complejas de sistemas complejos a los investigadores y autoridades de política. Aparte de los desafíos, los MBA son complementos promisorios de la cosecha actual de modelos macroeconómicos, en especial cuando dan sentido a los tipos de movimientos macroeconómicos extremos que el mundo ha presenciado en la última década.

\section{REFERENCIAS BIBLIOGRÁFICAS}

Aad, G., Abajyan, T. et al. (2012). Observation of a new particle in the search for the Standard Model Higgs boson with the ATLAS detector at the LHC. Physics Letters B, 716(1), 1-29. 
Abbott, B. P., Abbott, R. et al. (2016). Observation of gravitational waves from a binary black hole merger. Physical review letters, 116(6), 061102.

Abel, A. B. (1990). Asset prices under habit formation and catching up with the Joneses. NBER technical report. Cambridge, Mass.

Aikman, D., Galesic, M. et al. (2014). Taking uncertainty seriously: simplicity versus complexity in financial regulation. Bank of England financial stability paper 28. Londres.

Alfarano, S., Lux, T. y Wagner, F. (2005). Estimation of agent-based models: the case of an asymmetric herding model. Computational Economics, 26(1), 19-49.

Alfi, V., Cristelli, M. et al. (2009). Minimal agent based model for financial markets I. The European Physical Journal B, 67(3), 385-397.

Arber, T., Bennett, K. et al. (2015). Contemporary particle-in-cell approach to laser-plasma modelling. Plasma Physics and Controlled Fusion, 57(11), 113001.

Arinaminpathy, N., Kapadia, S. y May, R. (2012). Size and complexity in model financial systems. Proceedings of the National Academy of Sciences, 109(45), 18338-18343.

Arthur, W. B. (2006). Out-of-equilibrium economics and agent-based modeling. En L. Tesfatsion y K. Judd (eds.), Handbook of computational economics, v. 2 (pp. 1551-1564). Ámsterdam: Elsevier.

Ascari, G., Fagiolo, G. y Roventini, A. (2015). Fat-tail distributions and business-cycle models. Macroeconomic Dynamics, 19(02), 465-476.

Ashraf, Q., Gershman, B. y Howitt, P. (2017). Banks, market organization, and macroeconomic performance: an agent-based computational analysis. Journal of Economic Behavior E Organization, 135, 143-180.

Assenza, T., Brock, W. A. y Hommes, C. H. (2017). Animal spirits, heterogeneous expectations, and the amplification and duration of crises. Economic Inquiry, 55(1), 542-564.

Assenza, T., Gatti, D. D. y Grazzini, J. (2015). Emergent dynamics of a macroeconomic agent based model with capital and credit. Journal of Economic Dynamics and Control, 50, 5-28.

Assenza, T., Gatti, D. D. et al. (2016). Heterogeneous firms and international trade: The role of productivity and financial fragility. CESifo working paper series 5959. Múnich: CESifo Group.

Auclert, A. (2015). Monetary policy and the redistribution channel. 2015 meeting papers 381, Society for Economic Dynamics.

Ausloos, M., Miskiewicz, J. y Sanglier, M. (2004). The durations of recession and prosperity: does their distribution follow a power or an exponential law? Physica A: Statistical Mechanics and its Applications, 339(3), 548-558.

Avian Flu Working Group. (2006). The global economic and financial impact of an avian flu pandemic and the role of the IMF. Technical report, IMF.

Bagehot, W. (1873). Lombard Street: A description of the money market. Londres: Henry S. King and Co.

Baker, S. R., Bloom, N. y Davis, S. J. (2016). Measuring economic policy uncertainty. Quarterly Journal of Economics, 131(4), 1593-1636. 
Baptista, R., Farmer, J. D. et al. (2016). Macroprudential policy in an agent-based model of the UK housing market. Staff working paper 619. Londres: Bank of England.

Bardoscia, M., Battiston, S. et al. (2017). Pathways towards instability in financial networks. Nature Communications, 8(14416).

Bartelsman, E. J. y Doms, M. (2000). Understanding productivity: Lessons from longitudinal microdata. Journal of Economic literature, 38(3), 569-594.

Batten, S., Sowerbutts, R. et al. (2016). Let's talk about the weather: the impact of climate change on central banks. Staff working paper 603. Londres: Bank of England.

Battiston, S., Gatti, D. D. et al.. (2007). Credit chains and bankruptcy propagation in production networks. Journal of Economic Dynamics and Control, 31(6), 2061-2084.

Bernanke, B. (2004). The great moderation. Discurso en las reuniones de la Eastern Economic Association, 20 de febrero, Washington DC.

Bjørnland, H. C., Gerdrup, K. et al. (2012). Does forecast combination improve Norges Bank inflation forecasts? Oxford Bulletin of Economics and Statistics, 74(2), 163-179.

Blanchard, O. (2017). On the need for (at least) five classes of macro models, [https: //piie.com/blogs/realtime-economic-issues-watch/ need-least-five-classes-macro-models].

Bonabeau, E. (2002). Agent-based modeling: Methods and techniques for simulating human systems. Proceedings of the National Academy of Sciences, 99(supl. 3), 7280-7287.

Bottazzi, G. y Secchi, A. (2003). Common properties and sectoral specificities in the dynamics of US manufacturing companies. Review of Industrial Organization, 23(3-4), 217-232.

Bottazzi, G. y Secchi, A. (2006). Explaining the distribution of firm growth rates. RAND Journal of Economics, 37(2), 235-256.

Braun-M., K., Liu, Z. y Turrell, A. E. (2016). An agent-based model of dynamics in corporate bond trading. Staff working paper 592. Londres: Bank of England.

Brayton, F. y Tinsley, P. A. (1996). A guide to FRB/US: A macroeconomic model of the United States. FEDS paper 96-42. Washington DC. Federal Reserve Board.

Bronk, R. (2011). Uncertainty, modelling monocultures and the financial crisis. The business economist, 42(2), 5-18.

Bronk, R. y Jacoby, W. (2016). Uncertainty and the dangers of monocultures in regulation, analysis, and practice. MPIfG discussion paper 16/6, Colonia: Max Planck Institute.

Bulanov, S. y Khoroshkov, V. (2002). Feasibility of using laser ion accelerators in proton therapy. Plasma Physics Reports, 28(5), 453-456.

Burgess, S., Fernández-C., E. et al. (2013). The Bank of England's forecasting platform: COMPASS, MAPS, EASE and the suite of models. Staff working paper 471. Londres: Bank of England.

Burke, M., Hsiang, S. M. y Miguel, E. (2015). Global non-linear effect of temperature on economic production. Nature, 527(7577), 235-239. 
Burns, A. F. y Mitchell, W. C. (1946). Measuring business cycles. Cambridge, Mass: NBER.

Caiani, A., Godin, A. et al. (2016). Agent based-stock flow consistent macroeconomics: Towards a benchmark model. Journal of Economic Dynamics and Control, 69(C), 375-408.

Campbell, J. Y. y Mankiw, N. G. (1989). Consumption, income, and interest rates: Reinterpreting the time series evidence. NBER macroeconomics annual, 4, 185-216.

Card, D. y DellaVigna, S. (2013). Nine facts about top journals in economics. Journal of Economic Literature, 51(1), 144-161.

Carney, M. (2017). Reflecting diversity, choosing inclusion. Discurso Bank of England. Londres.

Carroll, C. D. (1997). Buffer-stock saving and the life cycle/permanent income hypothesis. Quarterly Journal of economics, 112(1), 1-55.

Carroll, C. D. (2009). Precautionary saving and the marginal propensity to consume out of permanent income. Journal of Mmonetary Economics, 56(6), 780-790.

Carroll, C. D. y Kimball, M. S. (1996). On the concavity of the consumption function. Econometrica, 64(4), 981-992.

Carter, N., Levin, S. et al. (2015). Modeling tiger population and territory dynamics using an agent-based approach. Ecological Modelling, 312, 347-362.

Castaldi, C. y Dosi, G. (2009). The patterns of output growth of firms and countries: Scale invariances and scale specificities. Empirical Economics, 37(3), 475-495.

Chakraborty, C. y Joseph, A. (2017). Machine learning at central banks. Staff working paper 674. Londres: Bank of England.

Chan, C. K. y Steiglitz, K. (2008). An agent-based model of a minimal economy. Department of Computer Science. Princeton: Princeton University.

Cincotti, S., Raberto, M. y Teglio, A. (2010). Credit money and macroeconomic instability in the agent-based model and simulator Eurace. Economics discussion papers 2010-4, Kiel Institute for the World Economy (IfW).

Colander, D., Goldberg, M. et al. (2009). The financial crisis and the systemic failure of the economics profession. Critical Review, 21(23), 249-267.

Colussi, T. (2018). Social ties in academia: A friend is a treasure. Review of Economics and Statistics, 100(1), 45-50.

Cooper, D. y Dynan, K. (2016). Wealth effects and macroeconomic dynamics. Journal of Economic Surveys, 30(1), 34-55.

Cutler, D. M., Poterba, J. M. y Summers, L. H. (1989). What moves stock prices? The Journal of Portfolio Management, 15(3), 4-12.

Davis, M., Efstathiou, G. et al. (1985). The evolution of large-scale structure in a universe dominated by cold dark matter. The Astrophysical Journal, 292, 371-394.

Dawid, H., Gemkow, S. et al. M. (2012). The eurace@ unibi model: An agent-based macroeconomic model for economic policy analysis. Bielefeld working papers in economics and management 05-2012. 
Dawid, H., Harting, P. y Neugart, M. (2014). Economic convergence: Policy implications from a heterogeneous agent model. Journal of Economic Dynamics and Control, 44(C), 54-80.

De Grauwe, P. (2010). Top-down versus bottom-up macroeconomics. CESifo Economic Studies, 56(4), 465-497.

Degli Atti, M. L. C., Merler, S. et al. (2008). Mitigation measures for pandemic influenza in Italy: an individual based model considering different scenarios. PloS one, 3(3), e1790.

Di Guilmi, C., Gallegati, M. y Ormerod, P. (2004). Scaling invariant distributions of firms' exit in OECD countries. Physica A: Statistical Mechanics and its Applications, 334(1), 267-273.

Doms, M. y Dunne, T. (1998). Capital adjustment patterns in manufacturing plants. Review of Economic Dynamics, 1(2), 409-429.

Dosi, G. (2007). Statistical regularities in the evolution of industries. A guide through some evidence and challenges for the theory. En F. Malerba y S. Brusoni (eds.), Perspectives on innovation (pp.153-186). Nueva York: Cambridge University Press.

Dosi, G., Fagiolo, G. et al. (2015). Fiscal and monetary policies in complex evolving economies. Journal of Economic Dynamics and Control, 52(C), 166-189.

Dosi, G., Fagiolo, G. y Roventini, A. (2010). Schumpeter meeting Keynes: A policy-friendly model of endogenous growth and business cycles. Journal of Economic Dynamics and Control, 34(9), 1748-1767.

Elmendorf, D. W. et al. (1996). The effect of interest-rate changes on household saving and consumption: A survey. FEDS paper 96-27. Washington DC. Federal Reserve Board.

Epstein, J. M. (1999). Agent-based computational models and generative social science. Complexity, 4(5), 41-60.

Epstein, J. M. (2006). Remarks on the foundations of agent-based generative social science. En L. Tesfatsion y K. Judd (eds.), Handbook of computational economics, v. 2 (pp.1585-1604). Ámsterdam: Elsevier.

Erlingsson, E. J., Teglio, A. et al. (2014). Housing market bubbles and business cycles in an agent-based credit economy. Economics: The Open-Access, Open-Assessment E-Journal, 8(2014-8), 1-42.

Ernest, N., Carroll, D. et al. (2016). Genetic fuzzy based artificial intelligence for unmanned combat aerial vehicle control in simulated air combat missions. Journal of Defense Management, 6(144), 2167-0374.

Estrella, A. y Fuhrer, J. C. (2002). Dynamic inconsistencies: Counterfactual implications of a class of rational-expectations models. American Economic Review, 92(4), 1013-1028.

Ezrachi, A. y Stucke, M. (2016). Virtual competition. The promise and perils of algorithmic-driven economy. Cambridge, Mass.: Harvard University Press.

Fagiolo, G., Napoletano, M. y Roventini, A. (2008). Are output growthrate distributions fat-tailed? Some evidence from OECD countries. Journal of Applied Econometrics, 23(5), 639-669.

Fagiolo, G. y Roventini, A. (2012). Macroeconomic policy in DSGE and agent-based models. Revue de l'OFCE, 5(124), 67-116. 
Fagiolo, G. y Roventini, A. (2017). Macroeconomic policy in DSGE and agent-based models Redux: New developments and challenges ahead. Journal of Artificial Societies and Social Simulation, 20(1), 1-37.

Fair, R. C. (2012). Has macro progressed? Journal of Macroeconomics, $34(1), 2-10$.

Foos, D., Norden, L. y Weber, M. (2010). Loan growth and riskiness of banks. Journal of Banking E Finance, 34(12), 2929- 2940.

Fourcade, M., Ollion, E. y Algan, Y. (2015). The superiority of economists. Revista de Economía Institutional, 17(33), 13-43.

Franke, R. y Westerhoff, F. (2012). Structural stochastic volatility in asset pricing dynamics: Estimation and model contest. Journal of Economic Dynamics and Control, 36(8), 1193-1211.

Friedman, J., Hastie, T. y Tibshirani, R. (2001). The elements of statistical learning, v. 1. Nueva York: Springer.

Friedman, M. (1957). A theory of the consumption function. Princeton: Princeton University Press.

Fukac, M. y Pagan, A. (2006). Issues in adopting DSGE models for use in the policy process. Australian National University, CAMA working paper 10: 2006.

Gabaix, X. (2011). The granular origins of aggregate fluctuations. Econometrica, 79(3), 733-772.

Gabaix, X. (2016). Behavioral macroeconomics via sparse dynamic programming. NBER technical report. Cambridge, Mass.

Gaffeo, E., Di Guilm. et al. (2012). On the mean/variance relationship of the firm size distribution: Evidence and some theory. Ecological Complexity, 11, 109-117.

Gai, P., Haldane, A. y Kapadia, S. (2011). Complexity, concentration and contagion. Journal of Monetary Economics, 58(5), 453-470.

Gai, P. y Kapadia, S. (2010). Contagion in financial networks. Proceedings of the Royal Society, 466(2120), 2401-2423.

Gatti, D. D. y Desiderio, S. (2015). Monetary policy experiments in an agent-based model with financial frictions. Journal of Economic Interaction and Coordination, 10(2), 265-286.

Geanakoplos, J., Axtell, R. et al. (2012). Getting at systemic risk via an agent-based model of the housing market. American Economic Review, $102(3), 53-58$.

Gibson, B. (2007). A multi-agent systems approach to microeconomic foundations of macro. Technical report working paper. Amherst: University of Massachusetts.

Gigerenzer, G. y Brighton, H. (2009). Homo heuristicus: Why biased minds make better inferences. Topics in cognitive science, 1(1), 107-143.

Gode, D. K. y Sunder, S. (1993). Allocative efficiency of markets with zero-intelligence traders: Market as a partial substitute for individual rationality. Journal of Political Economy, 101(1), 119-137.

Godley, W. y Lavoie, M. (2007). Monetary economics. Basingstoke: Palgrave Macmillan.

Gualdi, S., Tarzia, M. et al. (2015). Tipping points in macroeconomic agent-based models. Journal of Economic Dynamics and Control, 50, $29-61$. 
Guerini, M. y Moneta, A. (2017). A method for agent-based models validation. Journal of Economic Dynamics and Control, 82(C), 125-141.

Guerini, M., Napoletano, M. y Roventini, A. (2016). No man is an island: The impact of heterogeneity and local interactions on macroeconomic dynamics, [https://ssrn.com/abstract=2787164].

Guvenen, F. (2011). Macroeconomics with heterogeneity: A practical guide. NBER technical report. Cambridge, Mass.

Haldane, A. G. (2016). The dappled world. Discurso, Bank of England. Londres.

Haldane, A. G. y Madouros, V. (2012). E1 perro y el frisbee. Revista de Economia Institutional, 14(27), 13-56.

Haldane, A. G. y May, R. M. (2011). Systemic risk in banking ecosystems. Nature, 469(7330), 351-355.

Hamermesh, D. S. (2013). Six decades of top economics publishing: Who and how? Journal of Economic Literature, 51(1), 162-172.

Hausman, D. M. (1992). The inexact and separate science of economics. Gateshead, UK: Cambridge University Press.

Heathcote, J. (2005). Fiscal policy with heterogeneous agents and incomplete markets. Review of Economic Studies, 72(1), 161-188.

Heppenstall, A. J., Crooks, A. T. et al. (2011). Agent-based models of geographical systems. Dordrecht: Springer.

Hills, S., Thomas, R. y Dimsdale, N. (2016). Three centuries of data version 2.3, [http: //www.bankofengland.co. uk/research/Pages/onebank/threecenturies.aspx].

Hommes, C. H. (2006). Heterogeneous agent models in economics and finance. En L. Tesfatsion y K. Judd (eds.), Handbook of computational economics, v. 2 (pp. 1109-1186). Ámsterdam: Elsevier.

Hong, H. y Stein, J. C. (1999). A unified theory of underreaction, momentum trading, and overreaction in asset markets. Journal of finance, 54(6), 2143-2184.

Jacobs, J. A. (2014). In defense of disciplines: Interdisciplinarity and specialization in the research university. Chicago: University of Chicago Press.

Jaimovich, N. y Floetotto, M. (2008). Firm dynamics, markup variations, and the business cycle. Journal of Monetary Economics, 55(7), 1238-1252.

Jawadi, F. y Sousa, R. M. (2014). The relationship between consumption and wealth: A quantile regression approach. Revue d'économie politique, 124(4), 639-652.

Kaplan, G., Moll, B. y Violante, G. L. (2016). Monetary policy according to HANK. NBER technical report. Cambridge, Mass.

Keogh-B., M. R., Wren-L., S. et al. (2010). The possible macroeconomic impact on the UK of an influenza pandemic. Health economics, 19(11), 1345-1360.

Keynes, J. M. (1924). Alfred Marshall, 1842-1924. Economic Journal, 34(135), 311-372.

Keynes, J. M. (1936). General theory of employment, interest and money. Londres: Palgrave Macmillan.

Kindleberger, C. P. (2001). Manias, panics, and crashes: A history of financial crises. Hoboken, NJ: John Wiley \& Sons. 
Kirman, A. P. (1992). Whom or what does the representative individual represent? Journal of Economic Perspectives, 6(2), 117-136.

Knight, F. H. (2012). Risk, uncertainty and profit. North Chelmsford, Mass.: Courier Corporation.

Krugman, P. (2011). The profession and the crisis. Eastern Economic Journal, 37(3), 307-312.

Kumhof, M., Ranciere, R. y Winant, P. (2015). Inequality, leverage, and crises. American Economic Review, 105(3), 1217-1245.

Kuznets, S. y Murphy, J. T. (1966). Modern economic growth: Rate, structure, and spread, v. 2. New Haven: Yale University Press.

Kydland, F. E. y Prescott, E. C. (1982). Time to build and aggregate fluctuations. Econometrica, 50(6), 1345-1370.

Laeven, L. y Valencia, F. (2013). Systemic banking crises database. IMF Economic Review, 61(2), 225-270.

Lamperti, F., Dosi, G. et al. (2017a). Faraway, so close: Coupled climate and economic dynamics in an agent-based integrated assessment model. Sciences Po OFCE working paper 10.

Lamperti, F., Roventini, A. y Sani, A. (2017b). Agent-based model calibration using machine learning surrogates. Paper 1703.10639, arXiv.org.

Leary, M. T. (2009). Bank loan supply, lender choice, and corporate capital structure. Journal of Finance, 64(3), 1143-1185.

Leibo, J. Z., Zambaldi, V. et al. (2017). Multi-agent reinforcement learning in sequential social dilemmas. DeepMind working paper, Londres.

Leijonhufvud, A. (2000). Macroeconomic instability and coordination: Selected essays. Cheltenham: Edward Elgar.

Lengnick, M. (2013). Agent-based macroeconomics: A baseline model. Journal of Economic Behavior E Organization, 86, 102-120.

Leombruni, R. y Richiardi, M. (2005). Why are economists sceptical about agent-based simulations? Physica A: Statistical Mechanics and its Applications, 355(1), 103-109.

Linde, J., Smets, F. y Wouters, R. (2016). Challenges for central banks' macro models. En J. B. Taylor y H. Uhlig (eds.), Handbook of Macroeconomics, v. 2 (pp. 2185-2262). Ámsterdam: Elsevier.

Lind1, J. D., Amendt, P. et al. (2004). The physics basis for ignition using indirect-drive targets on the National Ignition Facility. Physics of Plasmas, 11(2), 339-491.

Lown, C. y Morgan, D. P. (2006). The credit cycle and the business cycle: new findings using the loan officer opinion survey. Journal of Money, Credit and Banking, 38(6), 1575-1597.

Lucas, R. E. (1972). Expectations and the neutrality of money. Journal of Economic Theory, 4(2), 103-124.

Lucas, R. E. (1976). Econometric policy evaluation: A critique. CarnegieRochester conference series on public policy, 1(1), 19-46.

Lucas, R. E. (1987). Models of business cycles, v. 26. Oxford: Basil Blackwell.

Lucas, R. E. y Sargent, T. J. (1979). After Keynesian macroeconomics. Quarterly Review, 3(2), 1-16.

Lux, T. y Marchesi, M. (1999). Scaling and criticality in a stochastic multi-agent model of a financial market. Nature, 397(6719), 498-500. 
Mendoza, E. G. y Terrones, M. E. (2012). An anatomy of credit booms and their demise. NBER technical report. Cambridge, Mass.

Metroplis, N. (1987). The beginning of the Monte Carlo method. Los Alamos Science, 15(548), 125-130.

Metropolis, N., Rosenbluth, A. W. et al. (1953). Equation of state calculations by fast computing machines. Journal of Chemical Physics, 21(6), 1087-1092.

Metropolis, N. y Ulam, S. (1949). The Monte Carlo method. Journal of the American Statistical Association, 44(247), 335-341.

Mikolov, T., Chen, K. et al. (2013). Efficient estimation of word representations in vector space. arXiv preprint arXiv: 13013781.

Minsky, H. P. (2008). Stabilizing an unstable economy, v. 1. Nueva York: McGraw Hill.

Muellbauer, J. y Murata, K. (2009). Consumption, land prices and the monetary transmission mechanism in Japan. Columbia University Academic Commons.

Muth, J. F. (1961). Rational expectations and the theory of price movements. Econometrica, 29(3), 315-335.

Napoletano, M., Roventini, A. y Sapio, S. (2006). Are business cycles all alike? A bandpass filter analysis of the Italian and US cycles. Rivista Italiana degli Economisti, 11(1), 87-118.

Nyman, R., Gregory, D. et al. (2015). News and narratives in financial systems: exploiting big data for systemic risk assessment. Bank of England staff working paper. Londres.

Page, S. E. (2008). The difference: How the power of diversity creates better groups, firms, schools, and societies. Princeton: Princeton University Press.

Popoyan, L., Napoletano, M. y Roventini, A. (2016). Taming macroeconomic instability: monetary and macro prudential policy interactions in an agent-based model. Journal of Economic Behavior E Organization, 134, 117-140.

Ravn, M. y Sterk, V. (2016). Macroeconomic fluctuations with HANK \& SAM: An analytical approach. Centre for Macroeconomics discussion papers 1633. University College London.

Reinhart, C. M. y Rogoff, K. S. (2009). The aftermath of financial crises. NBER technical report. Cambridge, Mass.

Romer, P. (2016). The trouble with macroeconomics. De próxima publicación en The American Economist.

Salle, I., Yildizoglu, M. y Senegas, M.-A. (2013). Inflation targeting in a learning economy: An MBA perspective. Economic Modelling, 34, 114-128.

Sands, P., Mundaca-S., C. y Dzau, V. J. (2016). The neglected dimension of global security - a framework for countering infectious-disease crises. New England Journal of Medicine, 374(13), 1281-1287.

Shaikh, A. (2016). Capitalism: Competition, conflict, crises. Nueva York: Oxford University Press.

Sherlock, M., Hill, E. et al. (2014). In-depth plasma-wave heating of dense plasma irradiated by short laser pulses. Physical review letters, 113(25); 255001-255036. 
Shiller, R. J. (2017). Narrative economics. NBER working paper 23075. Cambridge, Mass.

Silver, N. (2012). The signal and the noise: the art and science of prediction. Londres: Penguin.

Simon, H. A. (1959). Theories of decision-making in economics and behavioral science. American Economic Review, 49(3), 253-283.

Sinitskaya, E. y Tesfatsion, L. (2015). Macroeconomies as constructively rational games. Journal of Economic Dynamics and Control, 61(C), $152-182$.

Smets, F. y Wouters, R. (2003). An estimated dynamic stochastic general equilibrium model of the euro area. Journal of the European Economic Association, 1(5), 1123-1175.

Smith, N. (2014). Wall Street skips economics class. Bloomberg View, [https: //www.bloomberg.com/view/articles/2014-07-23/wall-streetskips-economics-class].

Solow, R. (2008). The state of macroeconomics. Journal of Economic Perspectives, 22(1), 243-246.

Sornette, D. (2014). Physics and financial economics (1776-2014): puzzles, Ising and agent-based models. Reports on progress in physics, 77(6), 062001-062014.

Souleles, N. S. (1999). The response of household consumption to income tax refunds. American Economic Review, 89(4), 947-958.

Spears, B. K., Munro, D. H. et al. (2015). Three-dimensional simulations of National Ignition Facility implosions: Insight into experimental observables a). Physics of Plasmas, 22(5), 056317.

Stern, N. (2016a). Current climate models are grossly misleading: Nicholas stern calls on scientists, engineers and economists to help policymakers by better modelling the immense risks to future generations, and the potential for action. Nature, 530(7591), 407-410.

Stern, N. (2016b). Economics: Current climate models are grossly misleading. Nature, 530(7591), 407-409.

Stern, N. H. (2007). The economics of climate change: the Stern review. Cambridge, UK: Cambridge University Press.

Stock, J. y Watson, M. (1999). Business cycle fluctuations in us macroeconomic time series. En J. B. Taylor y M. Woodford (eds.), Handbook of Macroeconomics, v, 1 (pp. 3-64). Âmsterdam: Elsevier.

Stock, J. H. y Watson, M. (2011). Dynamic factor models. En M. Clements y D. Hendry (eds.), Oxford Handbook on Economic Forecasting (pp. 35-60). Nueva York: Oxford University Press.

Stock, J. H. y Watson, M. W. (2006). Forecasting with many predictors. En G. Elliot. et al. (eds.), Handbook of economic forecasting, v. 1. (pp. 515-554). Ámsterdam: Elsevier.

Summers, L. H. (2002). Some skeptical observations on real business cycle theory. En B. Snowdon y H. Vane (eds.), A macroeconomics reader (pp. 389-394). Nueva York: Rutledge.

Tasoff, J., Mee, M. T. et al. (2015). An economic framework of microbial trade. PloS one, 10(7), e0132907.

Tesfatsion, L. (2002). Agent-based computational economics: Growing economies from the bottom up. Artificial life, 8(1), 55-82. 
Timmermann, A. (2006). Forecast combinations. En G. Elliot. et al. (eds.), Handbook of economic forecasting, v. 1 (pp. 135-196). Ámsterdam: Elsevier.

Turrell, A. (2016). Agent-based models: Understanding the economy from the bottom up. Bank of England quarterly bulletin series 2016Q4. Londres.

Turrell, A., Sherlock, M. y Rose, S. (2015a). Self-consistent inclusion of classical large-angle Coulomb collisions in plasma Monte Carlo simulations. Journal of Computational Physics, 299, 144-155.

Turrell, A., Sherlock, M. y Rose, S. (2015b). Ultrafast collisional ion heating by electrostatic shocks. Nature Communications, 6, 8905.

Tversky, A. y Kahneman, D. (1975). Judgment under uncertainty: Heuristics and biases. En D. Wendt y C. Vlek (eds.), Utility, probability, and human decision making (pp. 141-162). Dordrecht: Reidel Publishing.

Van Noorden, R. (2015). Interdisciplinary research by the numbers: an analysis reveals the extent and impact of research that bridges disciplines. Nature, 525(7569), 306-308.

Walde, K. y Woitek, U. (2004). R\&D expenditure in G7 countries and the implications for endogenous fluctuations and growth. Economics Letters, 82(1), 91-97.

Watts, D. J. (2002). A simple model of global cascades on random networks. Proceedings of the National Academy of Sciences, 99(9), 5766-5771.

Welfe, W. (2013). Macroeconometric models, v. 47. Berlín: Springer.

Wren-L., S. (2016a). More on stock-flow consistent models, [https: // mainlymacro.blogspot.co.uk/2016/09/more-on-stock-flow-consistentmodels.html].

Wren-L., S. (2016b). Unravelling the new classical counter revolution. Review of Keynesian Economics, 4(1), 20-35.

Wright, I. (2005). The duration of recessions follows an exponential not a power law. Physica A: Statistical Mechanics and its Applications, 345(3), 608-610.

Wuchty, S., Jones, B. F. y Uzzi, B. (2007). The increasing dominance of teams in production of knowledge. Science, 316(5827), 1036-1039.

Yegros-Y, A., Rafols, I. y D'Este, P. (2015). Does interdisciplinary research lead to higher citation impact? The different effect of proximal and distal interdisciplinarity. PloS one, 10(8), e0135095.

Yellen, J. L. et al. (2016, 14 de octubre). Macroeconomic research after the crisis: a speech at "The elusive 'great' recovery: Causes and implications for future business cycle dynamics". 60 conferencia económica anual patrocinada por el Reserve Bank of Boston. Boston, Mass.

Zarnowitz, V. (1985). Recent work on business cycles in historical perspective: A review of theories and evidence. Journal of Economic Literature, 23(2), 523-580. 